\title{
Multimodal categorization
}

\author{
Heinrich Bülthoff, Christian Wallraven
}




\section{Overview}

- The question of how the human brain "makes sense" of the sensory input it receives has been at the heart of cognitive and neuroscience research for the last decades.

- One of the most fundamental perceptual processes is categorization - the ability to compartmentalize knowledge for efficient retrieval.

- Recent advances in computer graphics and computer vision have made it possible to both produce highly realistic stimulus material for controlled experiments in life-like environments as well as to enable highly detailed analyses of the physical properties of realworld stimuli. 


\section{Research Philosophy}

- Study perception and action with stimuli as close as possible to the real world, using

- Computer Graphics to generate natural but well controlled stimuli of objects and scenes

- MPI Face Database (open access)

- faces.kyb.tuebingen.mpg.de

- vdb.kyb.tuebingen.mpg.de

- Database of High-Dynamic-Range Images (soon to come)

- Virtual Reality

to study perception and action in a closed loop

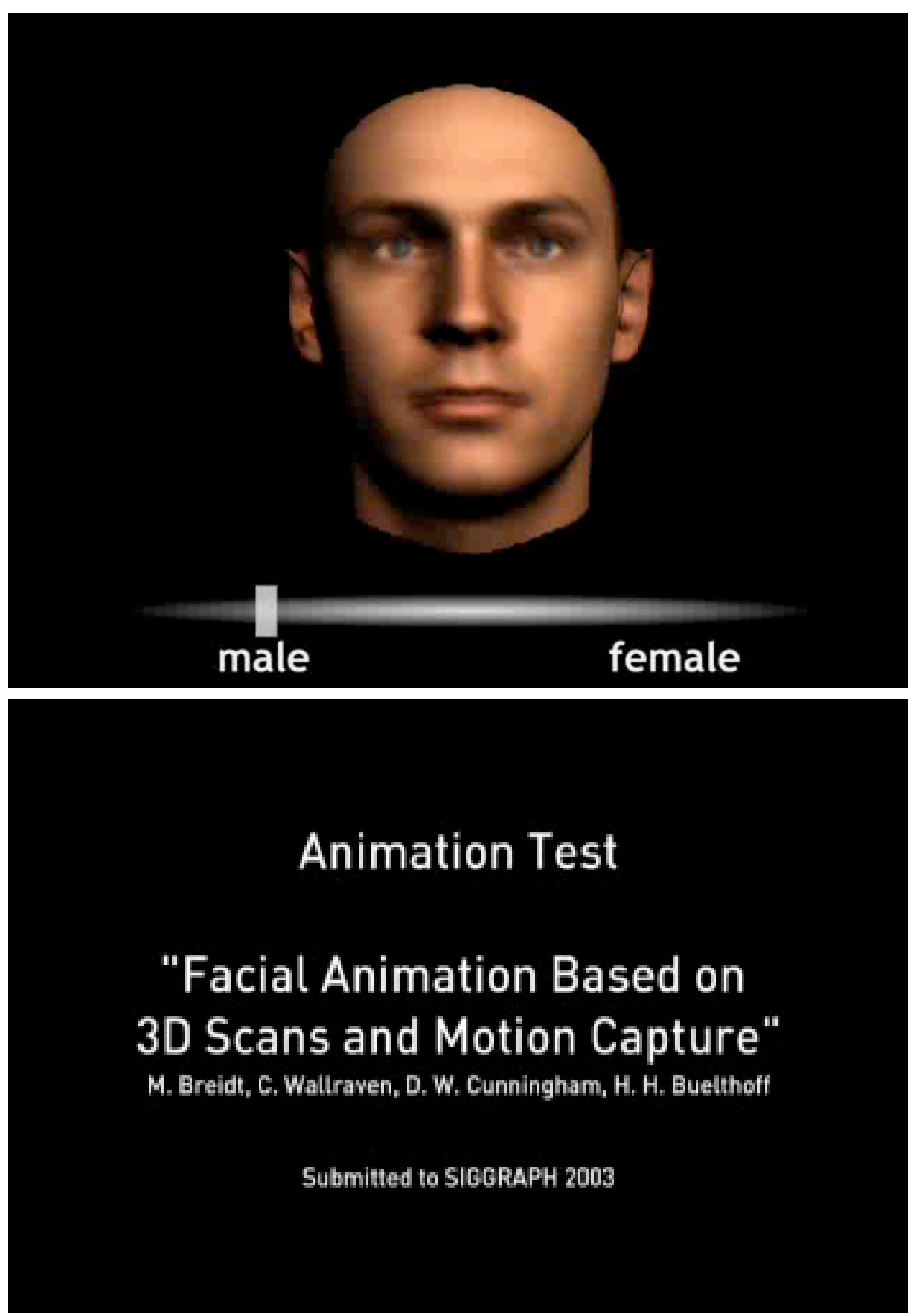




\section{Research Philosophy}

- Study perception and action with stimuli as close as possible to the real world, using

- Computer Graphics

to generate natural but well

controlled stimuli of objects and scenes

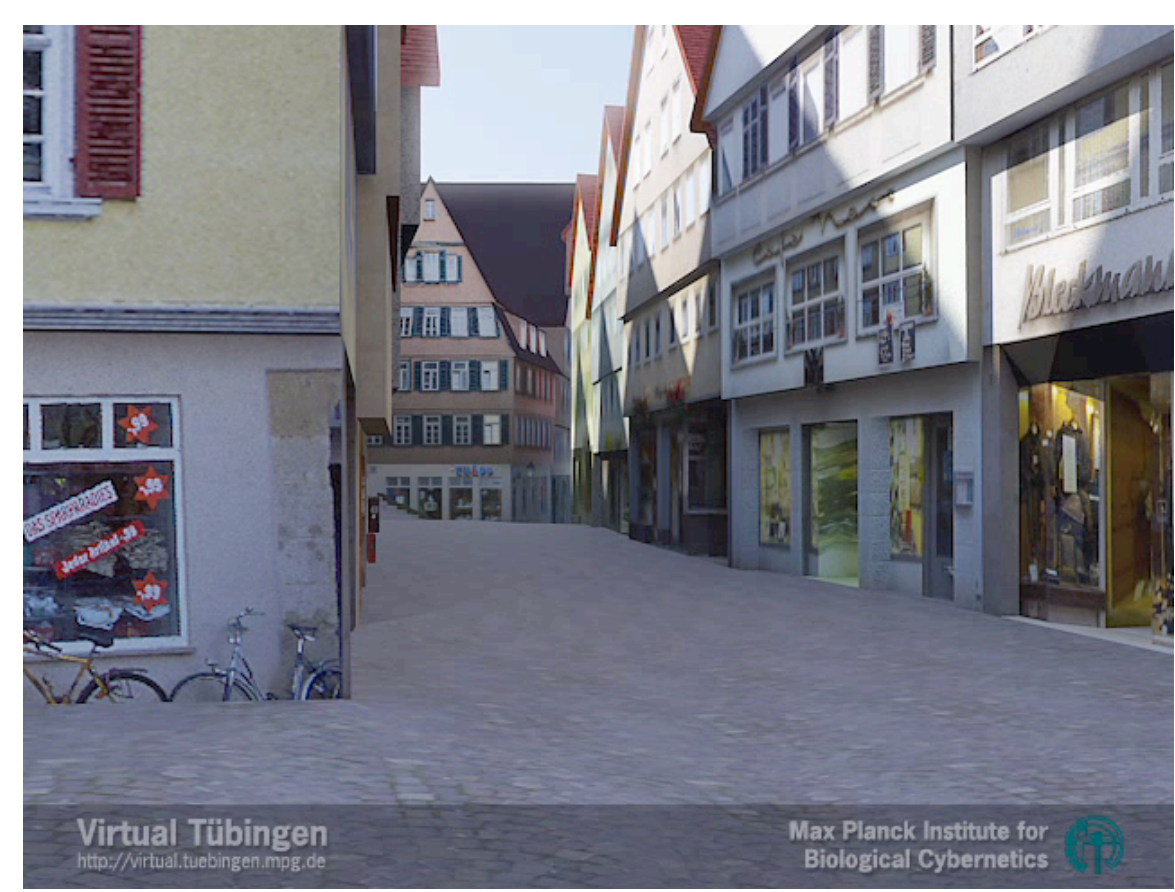

- Virtual Reality

- www.cyberneum.de

- motion simulators

- haptic simulators

- walking simulators

- immersive environments

- panoramic projections

- EU-projects: JAST, BACS, CyberWalk, Immersense, Wayfinding

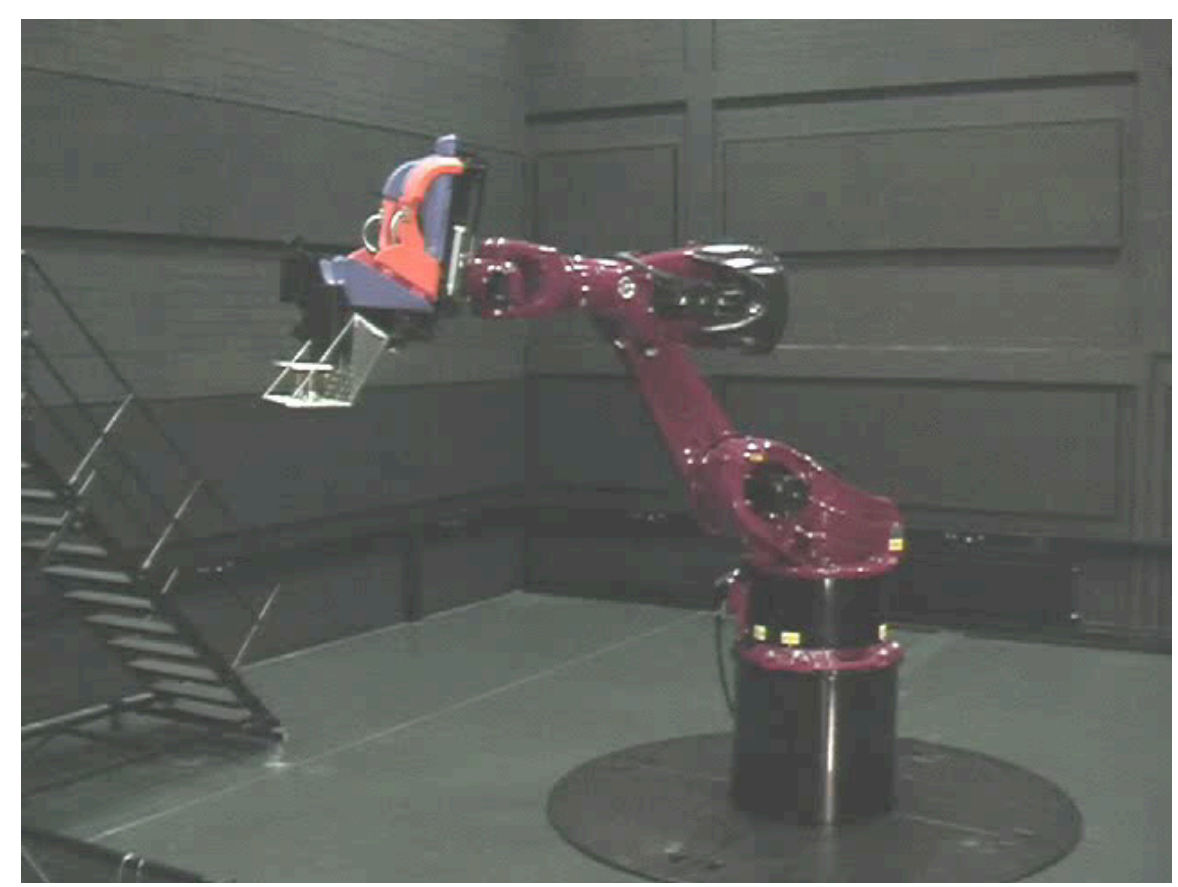




\section{Overview}

- In this talk, we will review some of the key challenges in understanding categorization from a combined cognitive and computational perspective:

- the need for spatio-temporal representations

- perception of material properties

- multi-modal/multi-sensory aspects of object categorization

- coupling of perception and action 


\section{Research Paradigm}

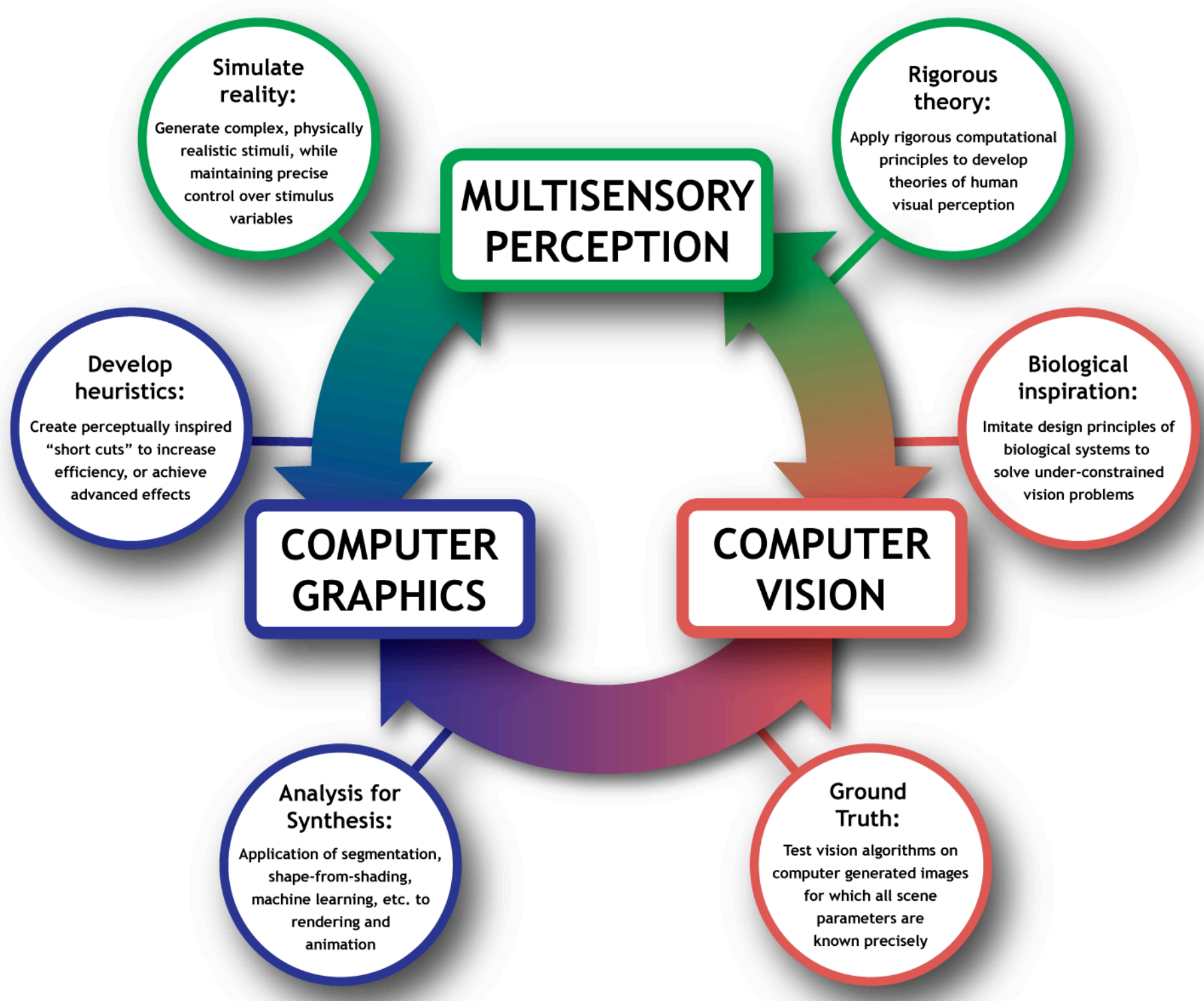




\section{Overview}

- The talk will focus on issues that so far have only started to be addressed but that are crucial for a deeper understanding of perceptual processes:

- the need for spatio-temporal representations

- perception of material properties

- multi-modal/multi-sensory aspects of object categorization

- coupling of perception and action 


\section{Representing objects: two models}

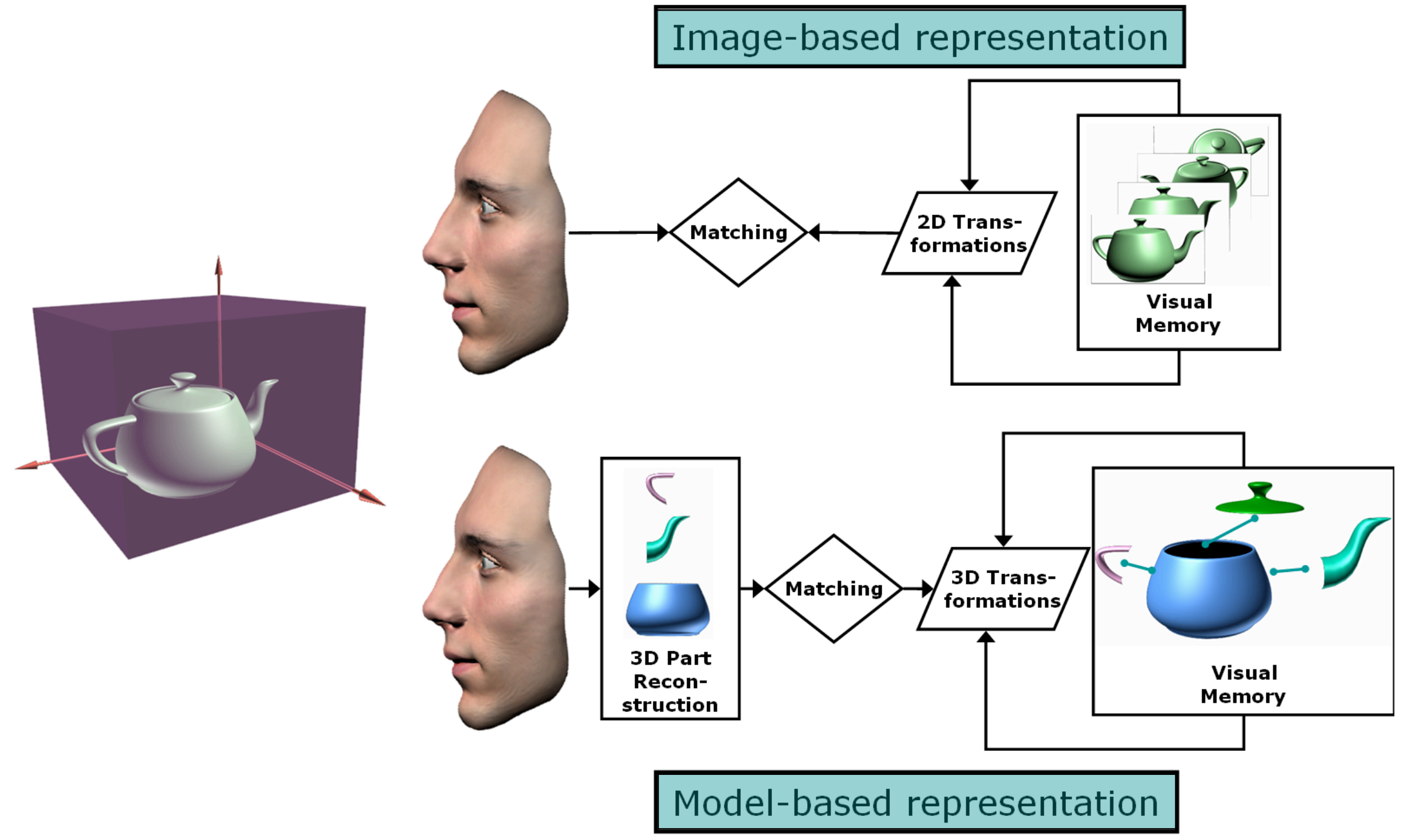




\section{Representing objects: image-based recognition}

Bülthoff and Edelman [PNAS, 1992]

- Recognition of novel objects depends on the viewing conditions $(\rightarrow$ image-based recognition)
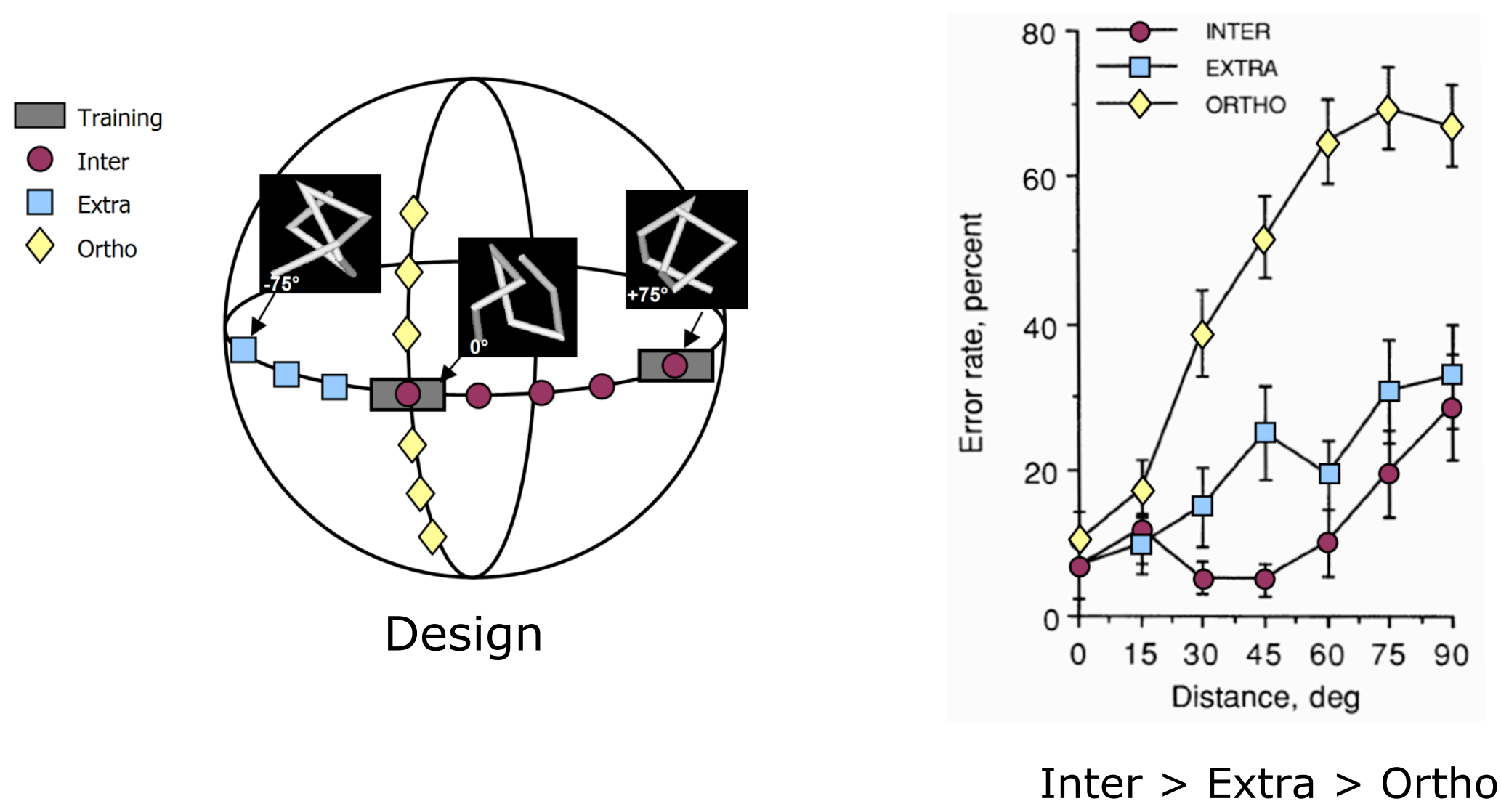


\section{Representing faces: image-based recognition}

Wallraven, Schwaninger, Schumacher, Bülthoff [BMCV, 2002]

- Recognition of novel and familiar objects depends on the viewing conditions ( $\rightarrow$ image-based recognition)
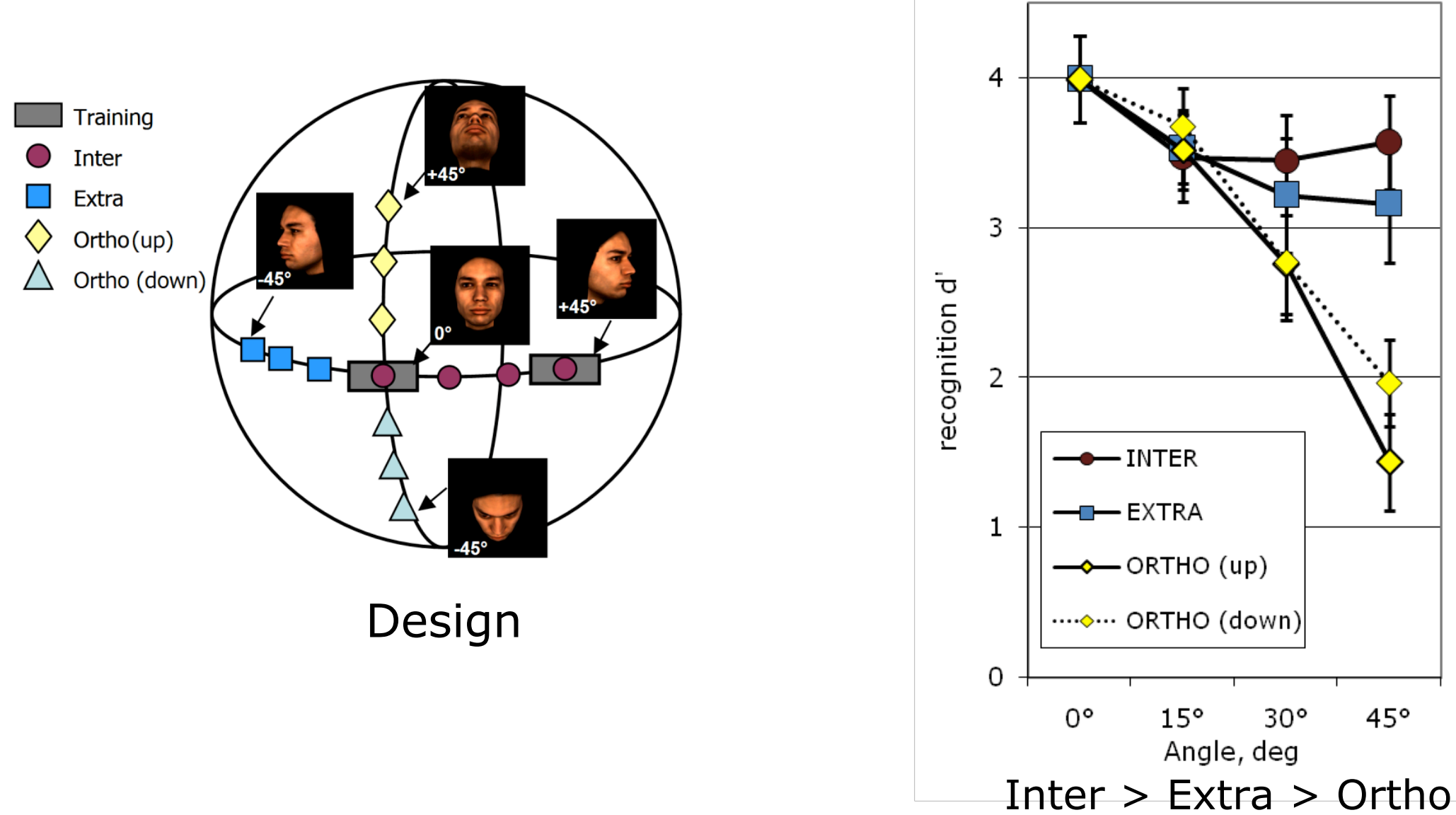


\section{The role of motion in recognition}

1. Familiar motion facilitates person identification

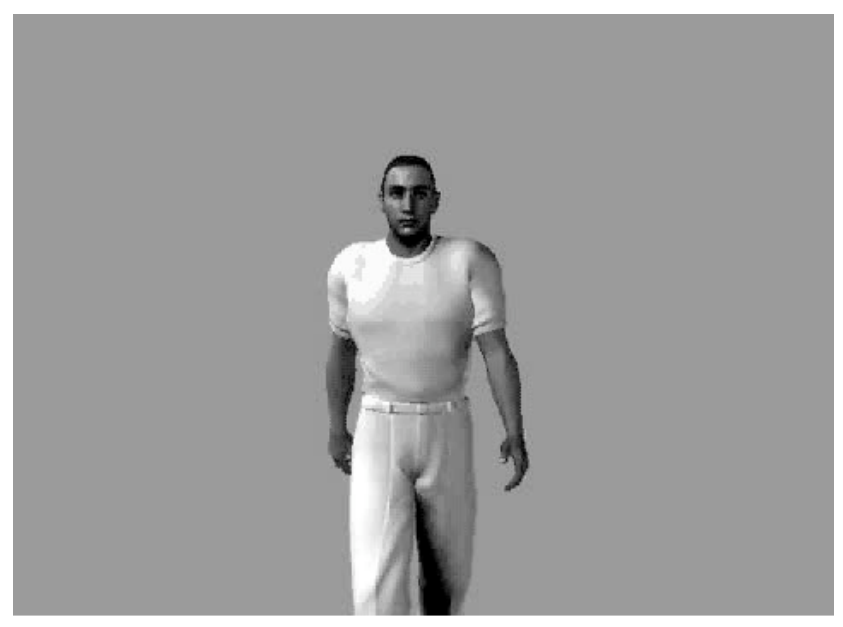

Pilz, Vuong, Bülthoff, Thornton [JEP: HPP, subm]
2. Motion facilitates human target detection

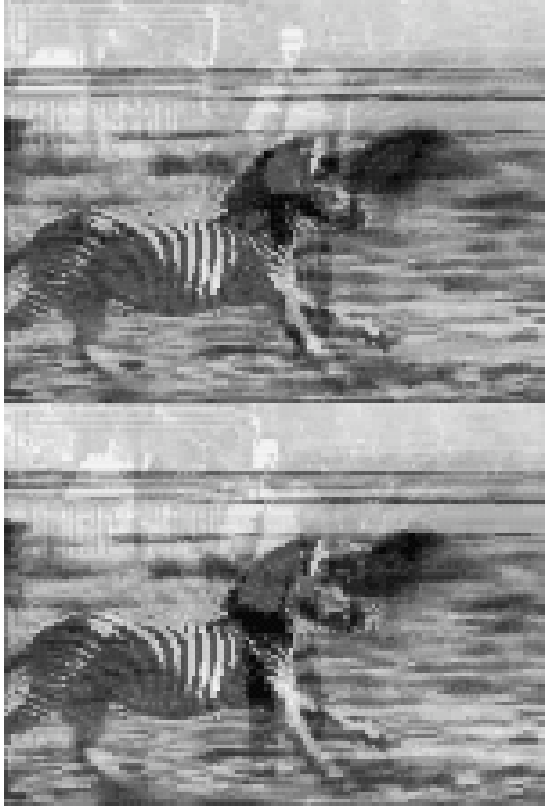

Vuong, Hof, Bülthoff, Thornton [Journal of Vision, 2006]
3. Non-rigid motion is encoded as identity cue

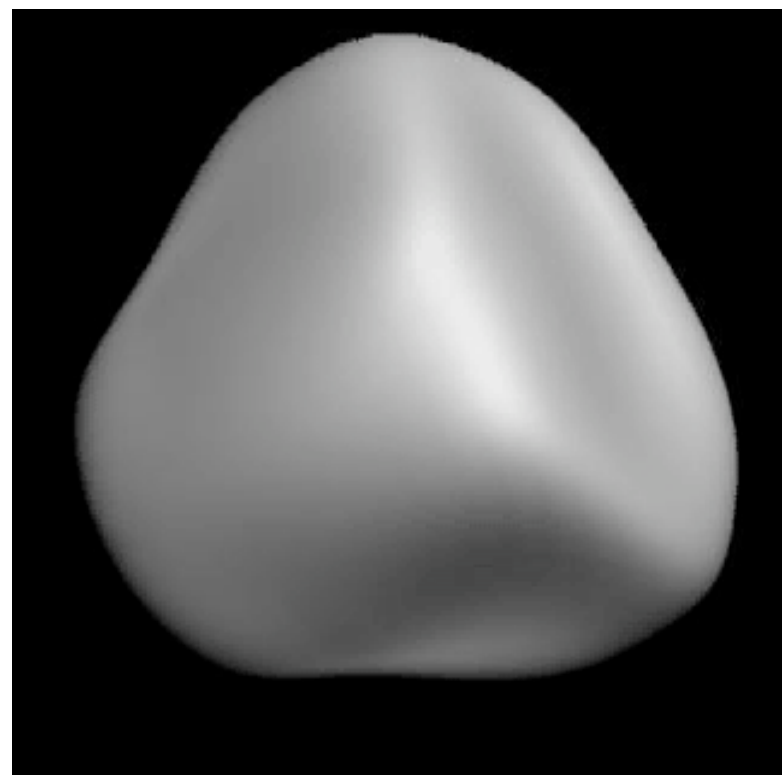

Chuang, Vuong, Thornton, Bülthoff [Visual Cognition, 2006] 


\section{Quick summary (Spatio-temporal representations)}

- Objects and faces are represented in an image-based fashion

- The temporal properties of objects play an important role during learning and recognition

- Object representations are spatio-temporal 


\section{Overview}

- In this talk, we will review some of the key challenges in understanding categorization from a combined cognitive and computational perspective:

- the need for spatio-temporal representations

- perception of material properties

- multi-modal/multi-sensory aspects of object categorization

- coupling of perception and action 


\section{Image-based material editing}

Kahn, Reinhard, Fleming, Bülthoff [SIGGRAPH, 2006]

- Goals:

- How do humans perceive materials?

- Ill-posed problem

- Can we exploit perceptual tricks to change materials in a photograph (without a 3D-model)?

- Methods:

- Crude 3D shape reconstruction using bilateral filter (dark means deep - SFS)

- Exploits generic viewpoint assumption as an image is consistent with many 3D models

- Simple background-inpainting for transparency

- Exploits masking

- weak model of refraction

- Results:

- Re-texturing

- Medium gloss to matte or glossy

- Opaque to transparent or translucent

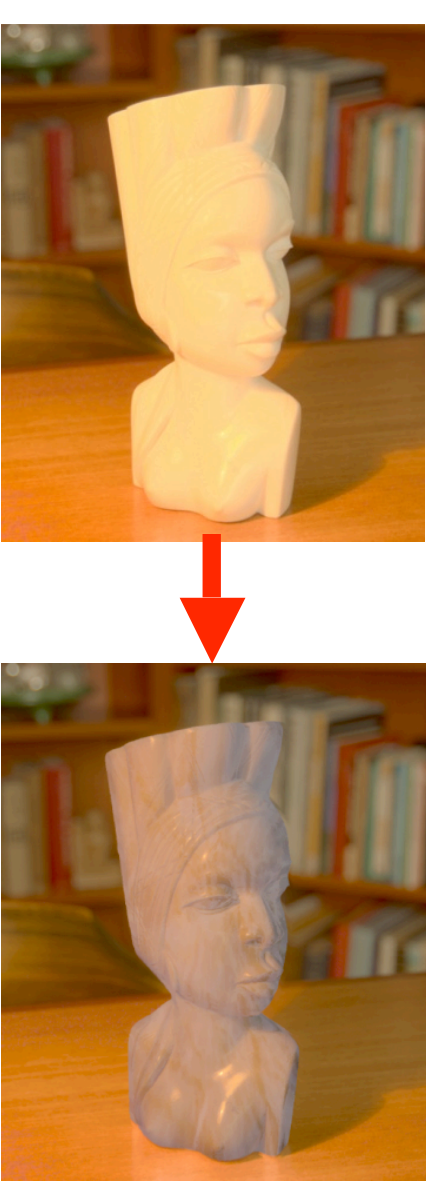

re-textured

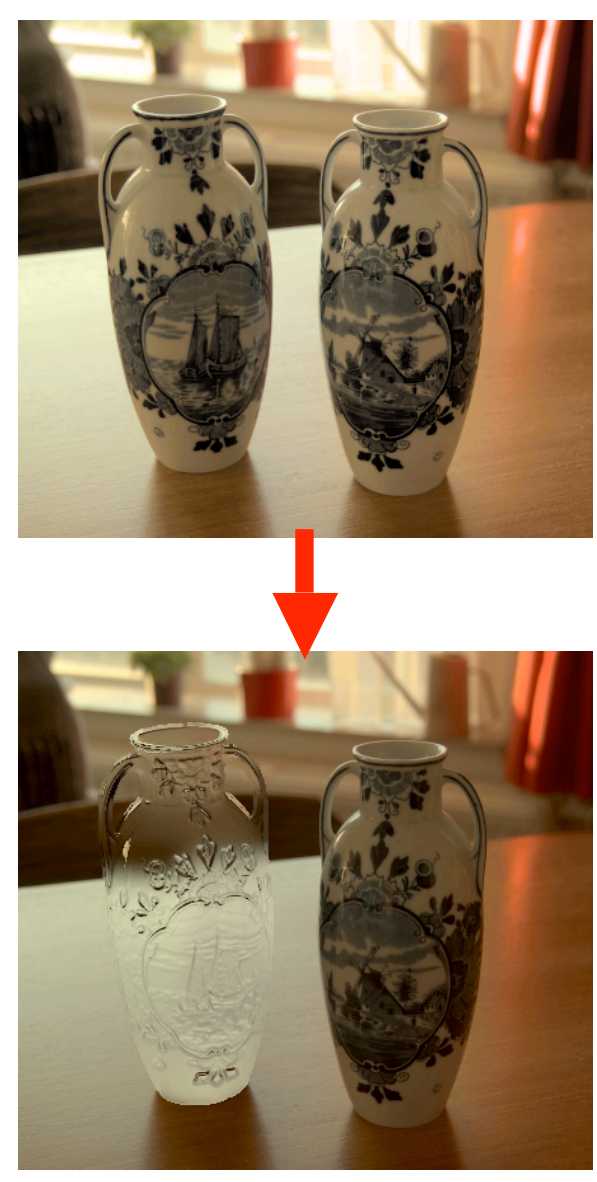

transparency 
Image-based material editing

Kahn, Reinhard, Fleming, Bülthoff [SIGGRAPH, 2006]

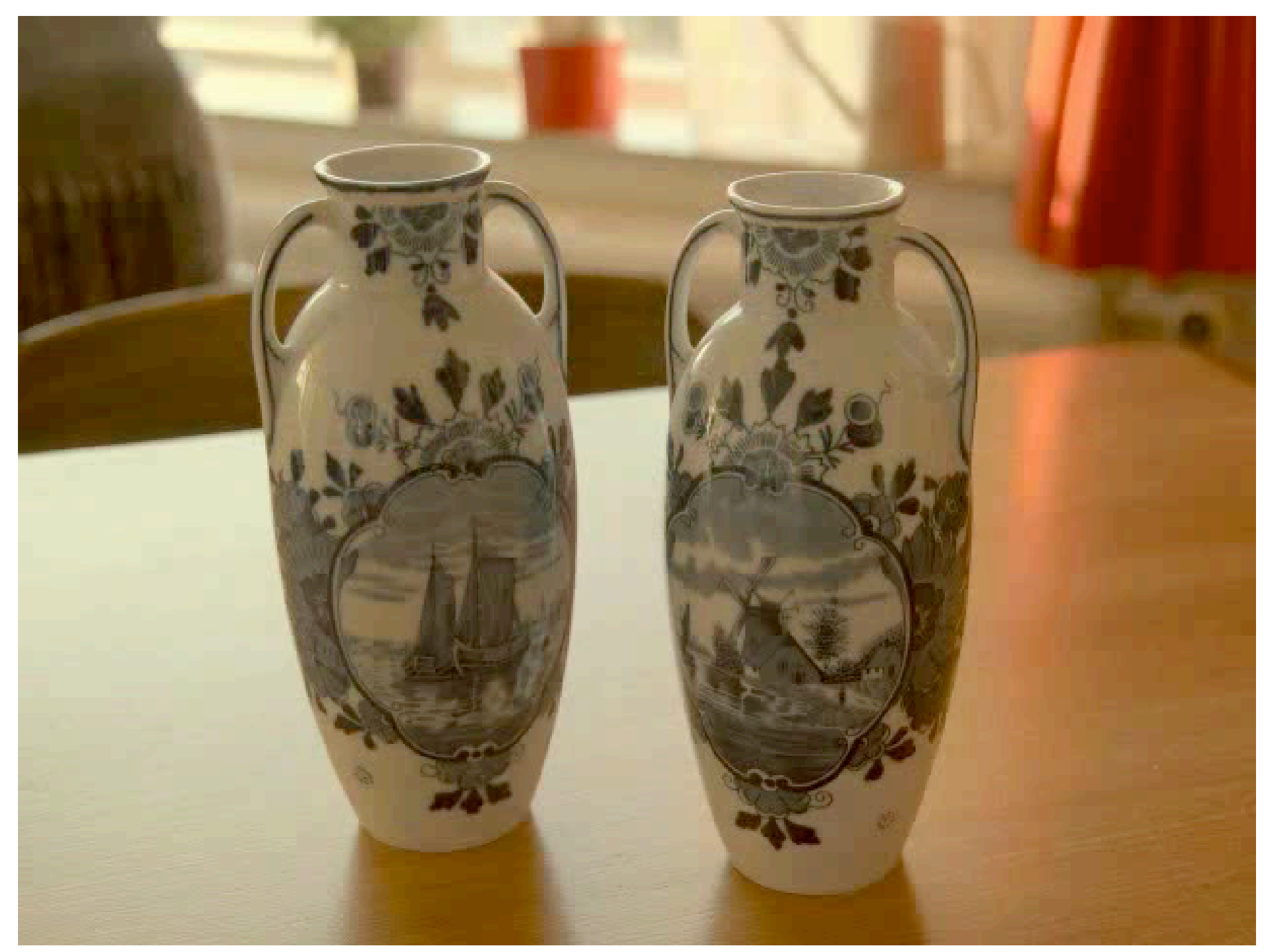




\section{Quick summary (Material Perception)}

- The brain does not use an inverse physics approach to perception

- Rather, the brain uses (complex) heuristics to estimate

- Material properties

- Shape

- By exploiting these heuristics one can create simple, but effective work-arounds to control these properties 


\section{Overview}

- In this talk, we will review some of the key challenges in understanding categorization from a combined cognitive and computational perspective:

- the need for spatio-temporal representations

- perception of material properties

- multi-modal/multi-sensory aspects of object categorization

- coupling of perception and action 


\section{Sensory integration}

- Humans act upon objects in order to interact with the world.

- Two studies addressed the following questions:

- Are object representations multi-modal?

- How can we teach artificial agents how to interact with the world?

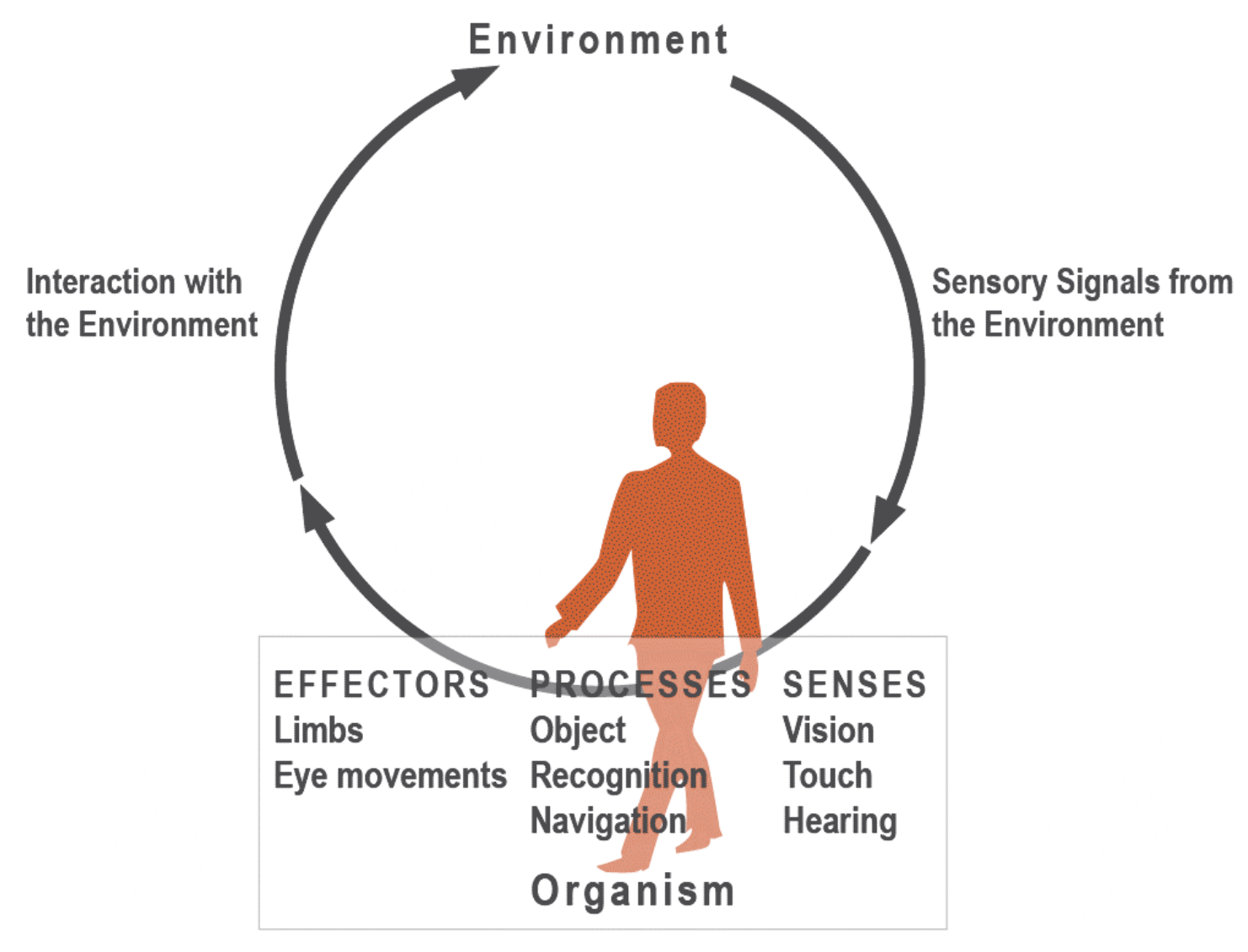




\section{Sensory integration}

- Humans act upon objects in order to interact with the world.

- Two studies addressed the following questions:

- Are object representations multi-modal?

- How can we teach artificial agents how to interact with the world?

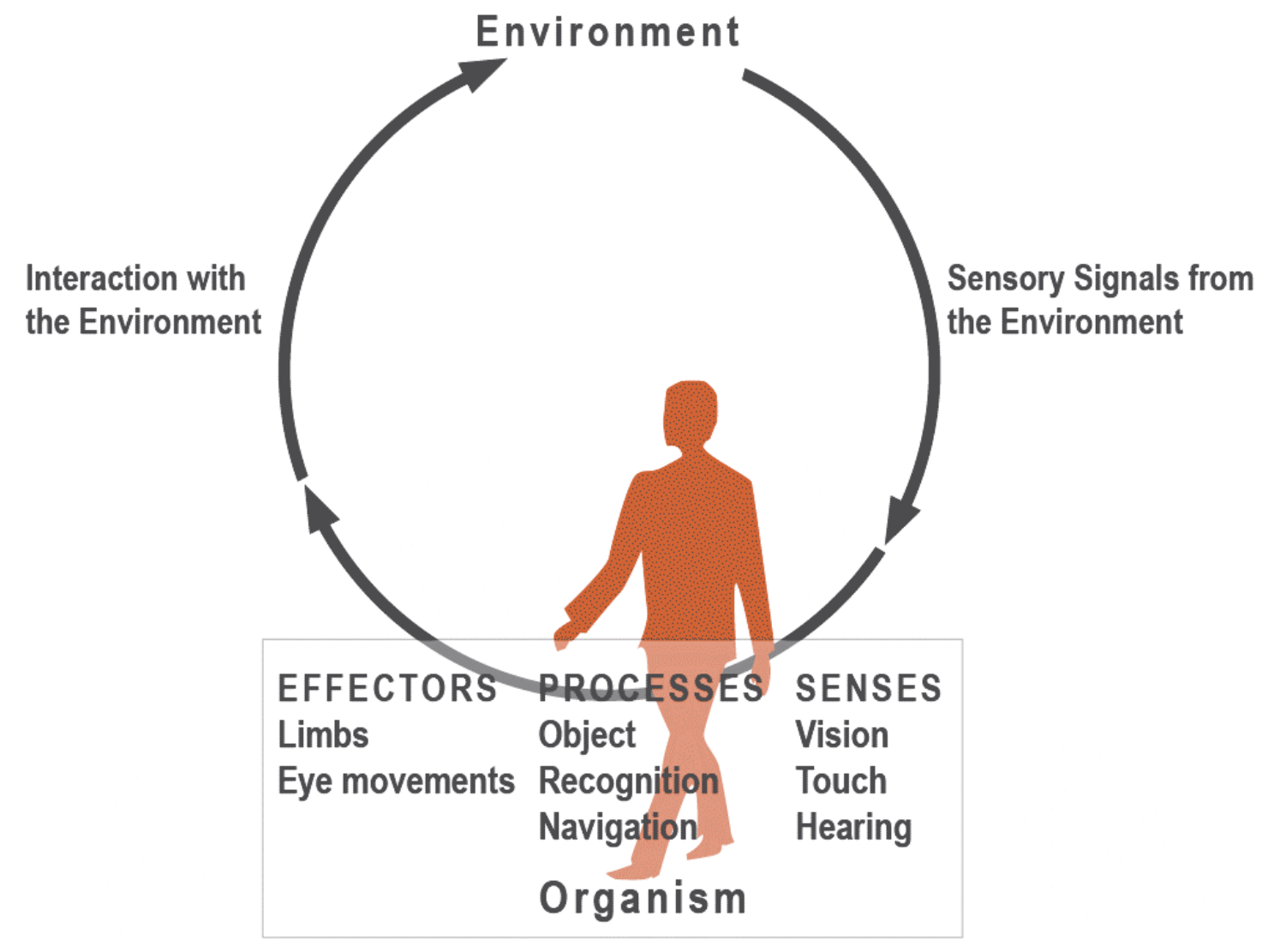


Multi-modal similarity and categorization of novel, 3D objects Cooke, Jäkel, Wallraven, Bülthoff [Neuropsychologia, 2007]

- Goal:

- Develop framework for understanding multi-sensory (visuo-haptic) object perception

- Methods:

- Controlled space of visuohaptic stimuli printed in 3D

- Multi-Dimensional-Scaling for finding perceptual space for haptic, visual and bimodal exploration
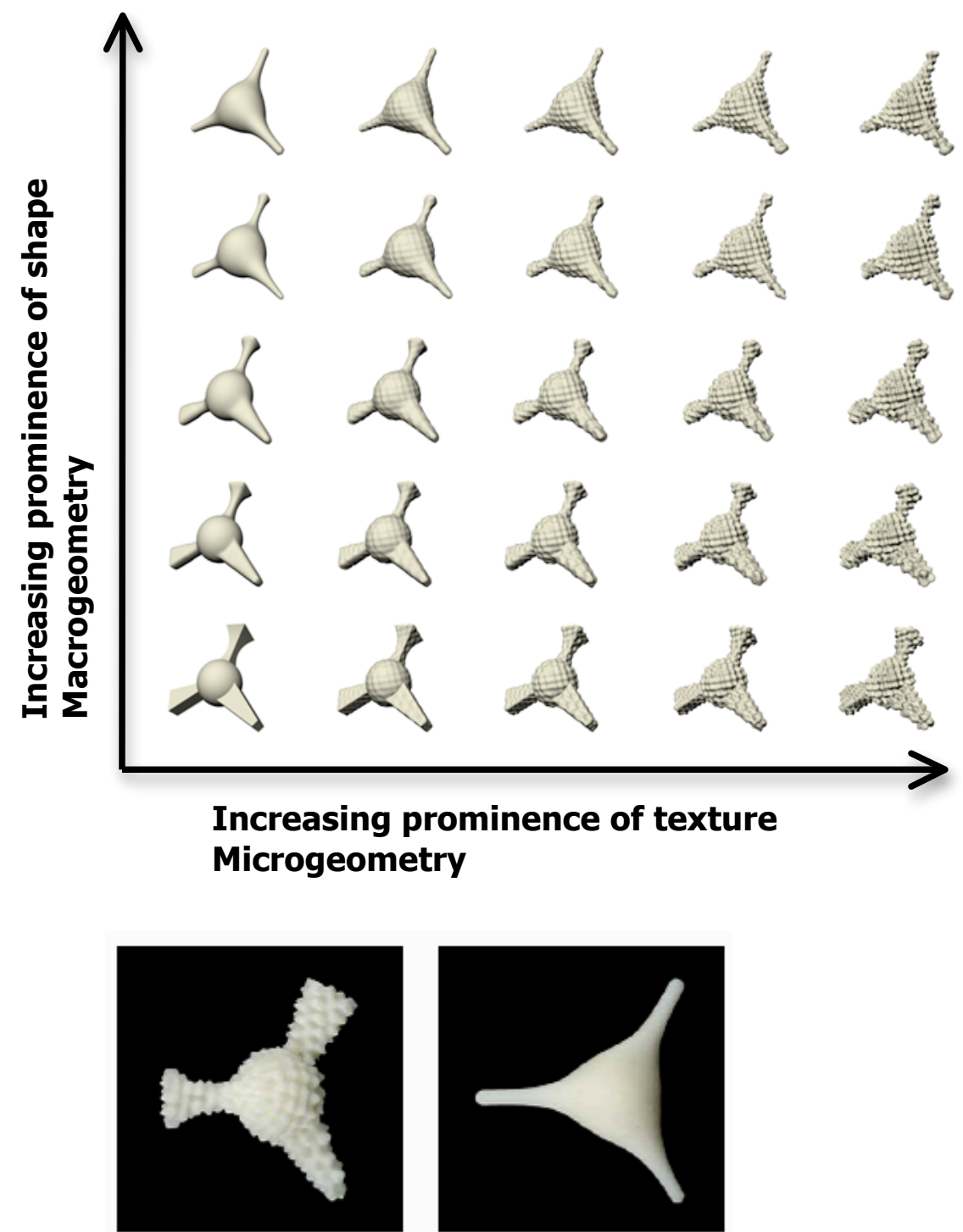

Photographs of printed 3D objects 
The tools: Parametrically-defined stimuli \& 3D printer Cooke, Jäkel, Wallraven, Bülthoff [Neuropsychologia, 2007]

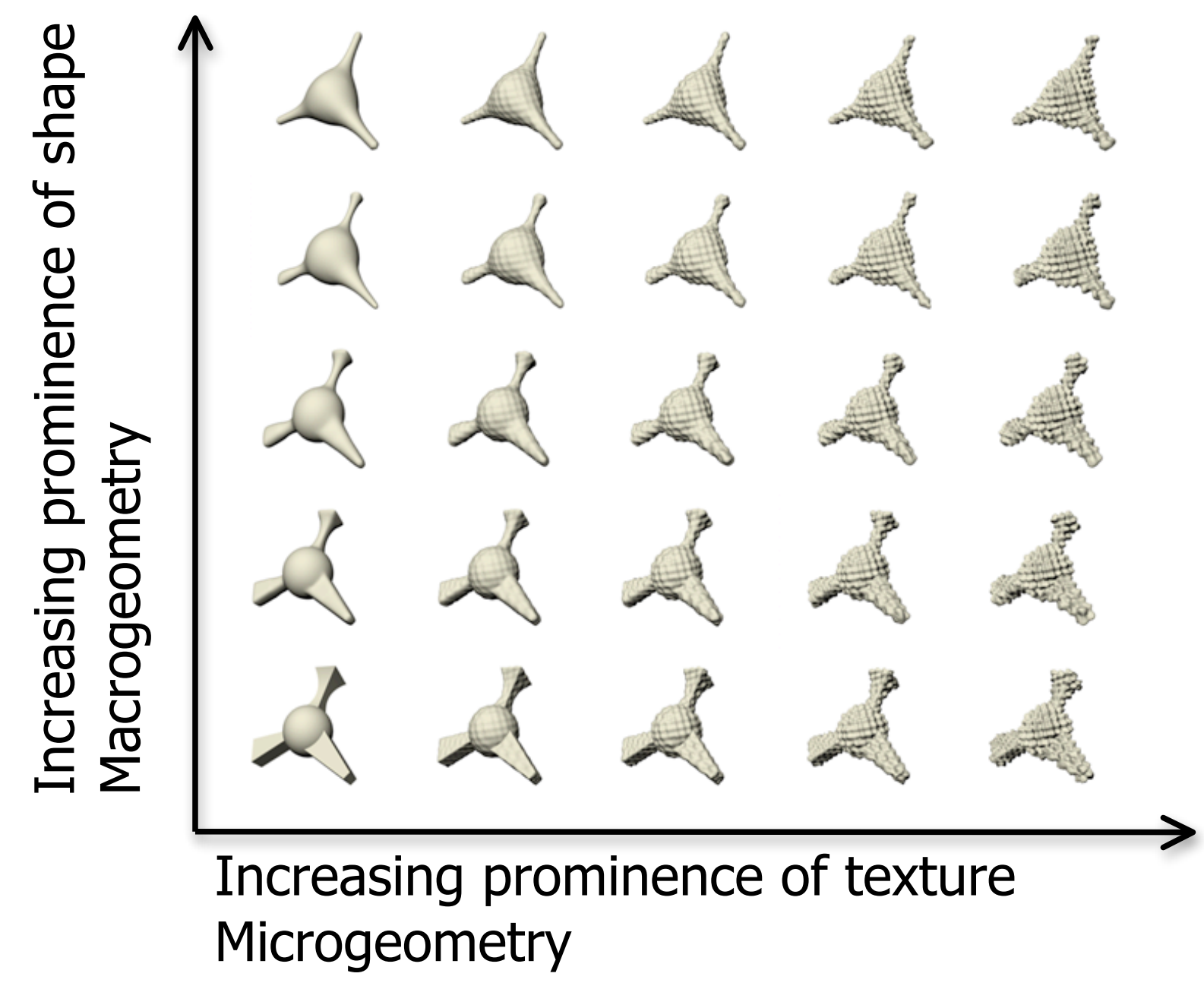

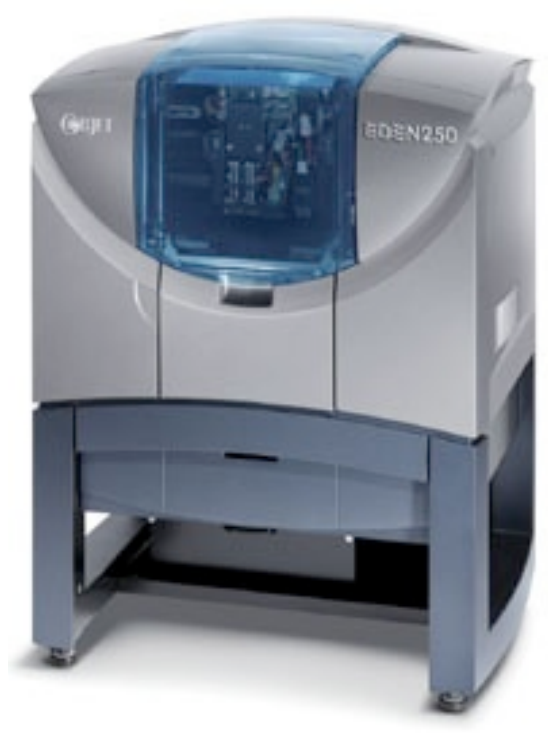

3D printer

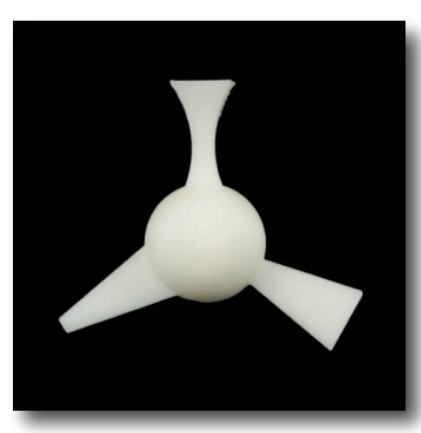

Printed object 
The experiment: Multi-sensory similarity

Cooke, Jäkel, Wallraven, Bülthoff [Neuropsychologia, 2007]
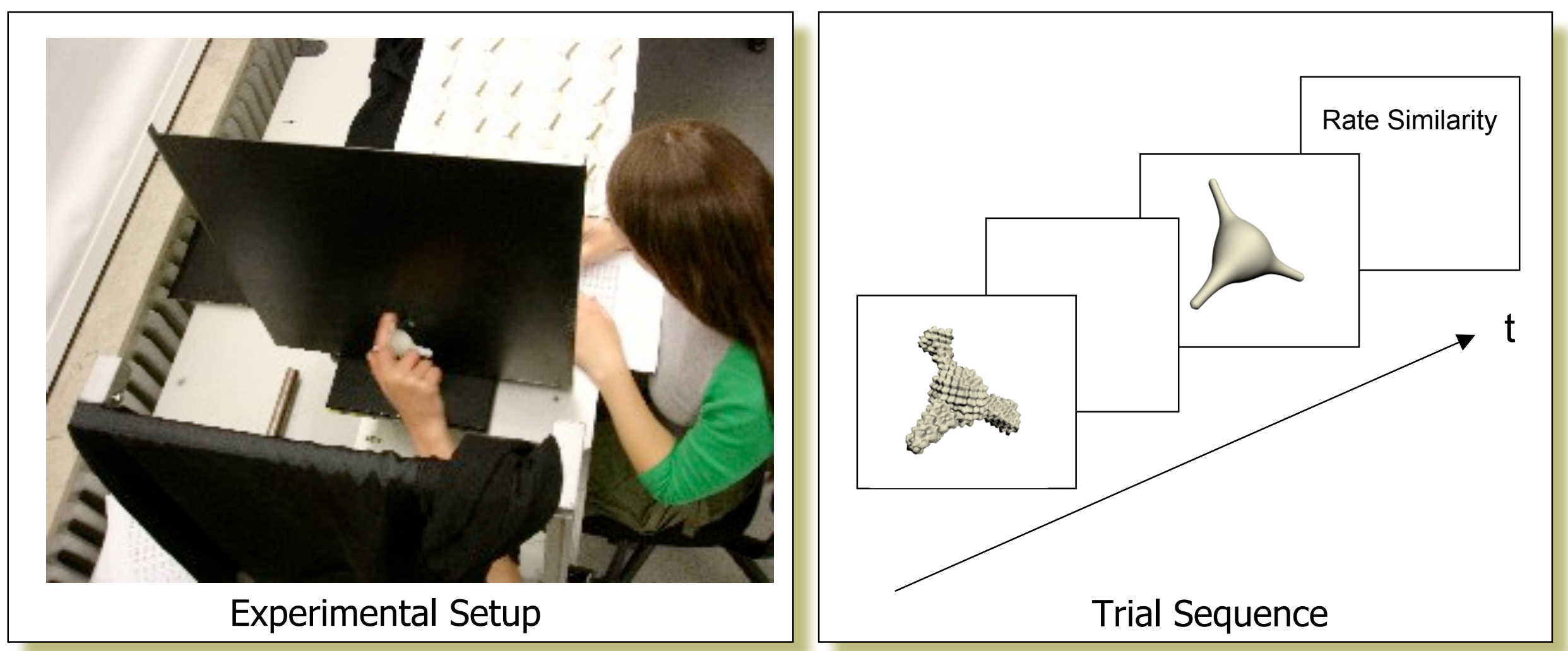

- 10 subjects $x 3$ conditions: Visual (V), Haptic (H), Visuohaptic (VH)

- Task : Similarity ratings 
Results: Modality Effects

Cooke, Jäkel, Wallraven, Bülthoff [Neuropsychologia, 2007]

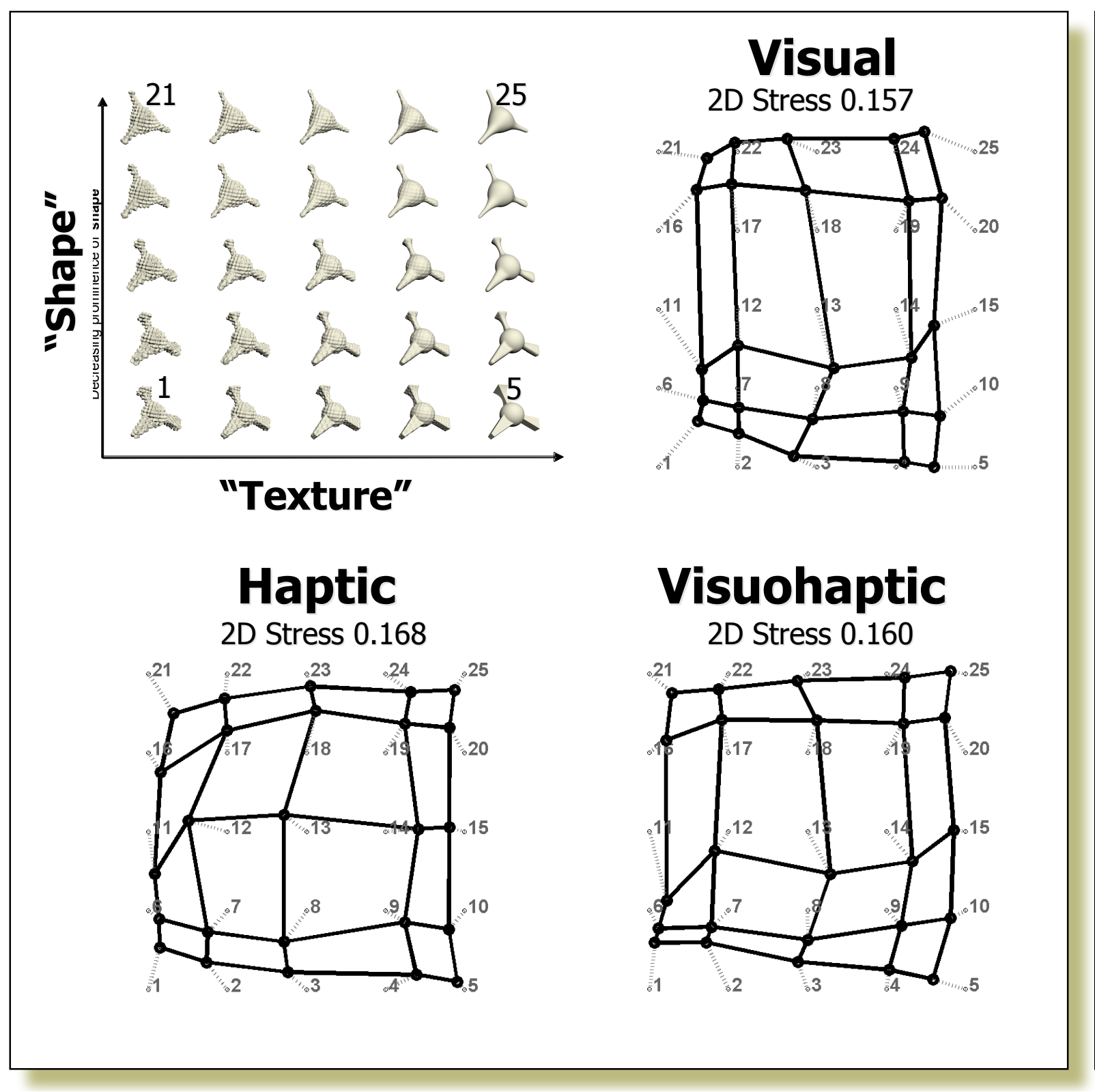

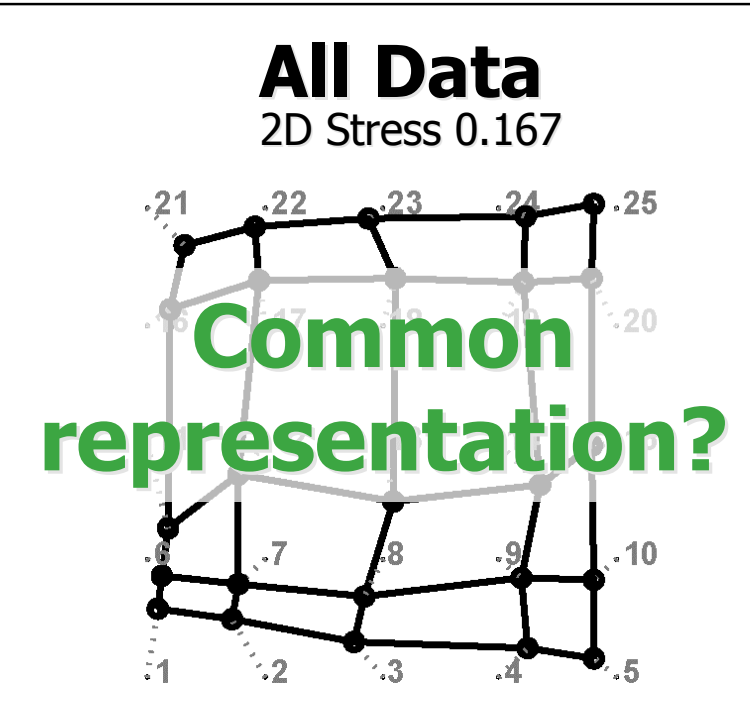

Relative Weights

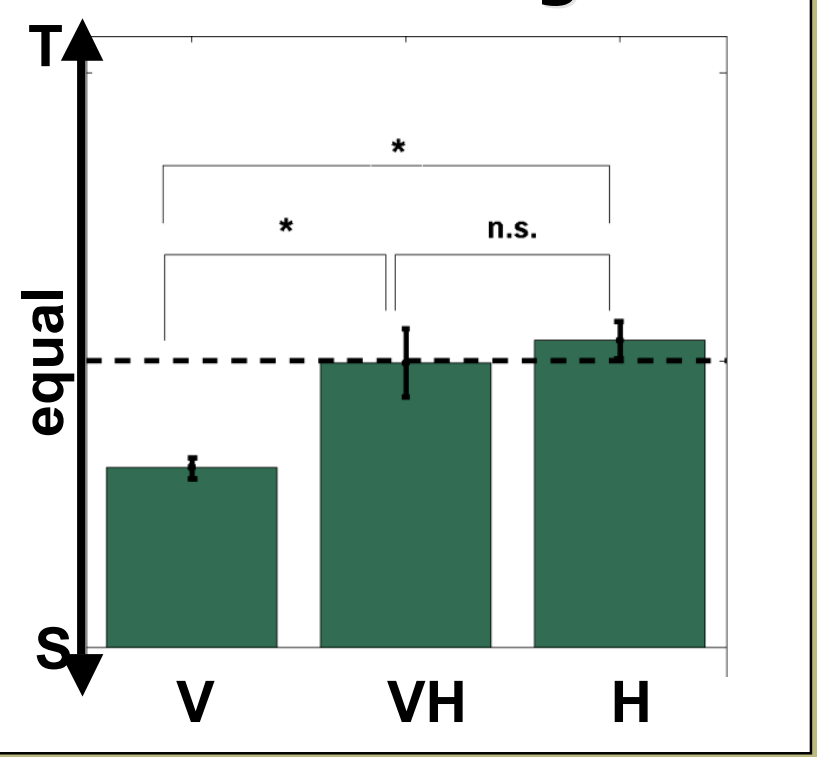




\section{Perceptual Feature Toolbox}

Wallraven, Cooke, Kannengießer [http://pft.homeunix.org/, 2007]

- Goal:

- Develop toolbox for perceptual feature validation

- Methods:

- 2D features from computer vision

- Pixel values, Edge Images, Gabor filter response, Visual Difference Predictor, Structural Similarity, Shape Context

- 3D features from computer graphics

- Vertex Coordinates, Vertex Count, Perimeter, Mean Local Curvature, Shape Histograms

- Results:

- Applied to Visuo-haptic similarity ratings:

- Most 2D features model visual similarity judgments well but were not able to model human haptic perception
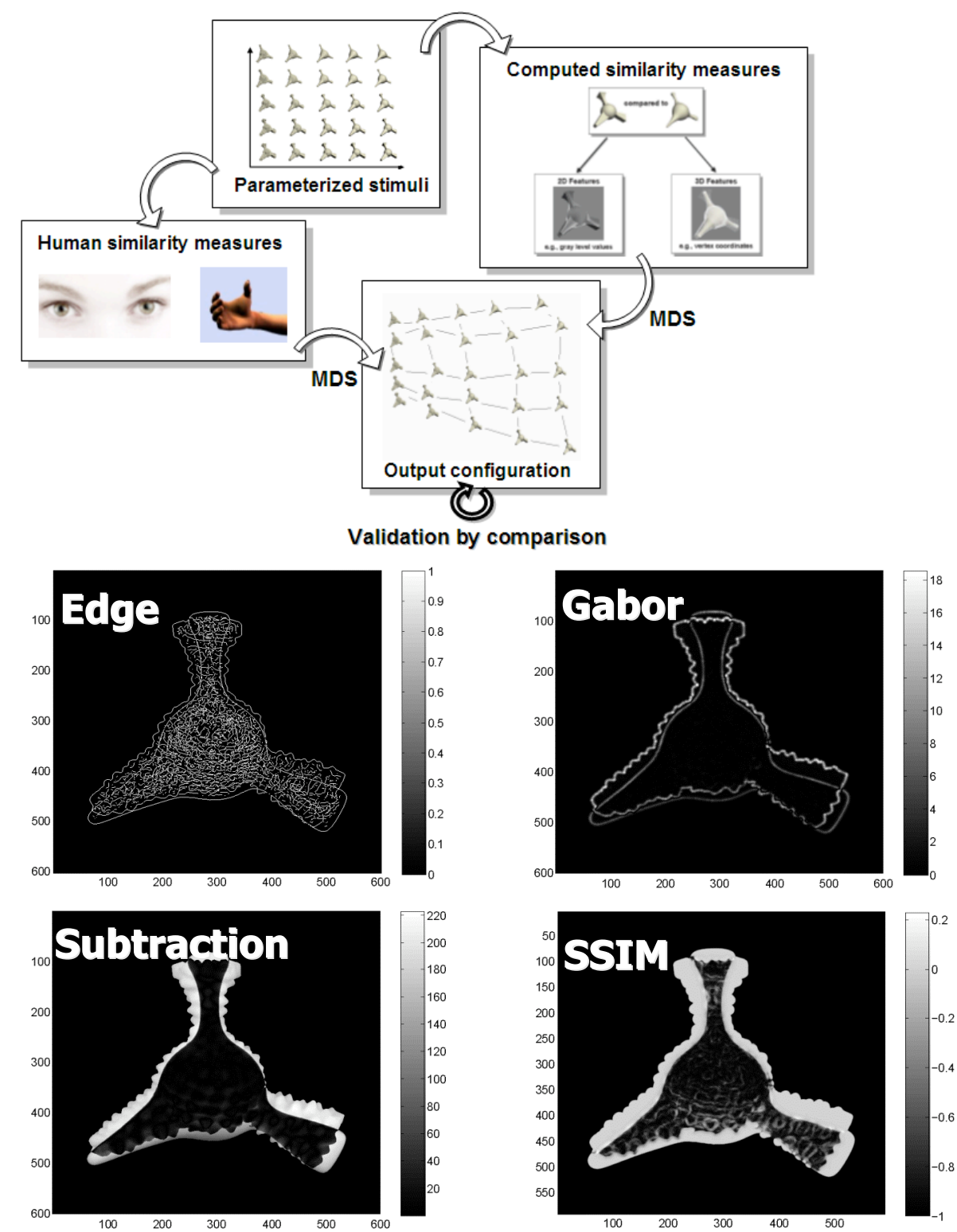


\section{Sensory integration}

- Humans act upon objects in order to interact with the world.

- Two studies addressed the following questions:

- Are object representations multi-modal?

- How can we teach artificial agents how to interact with the world?

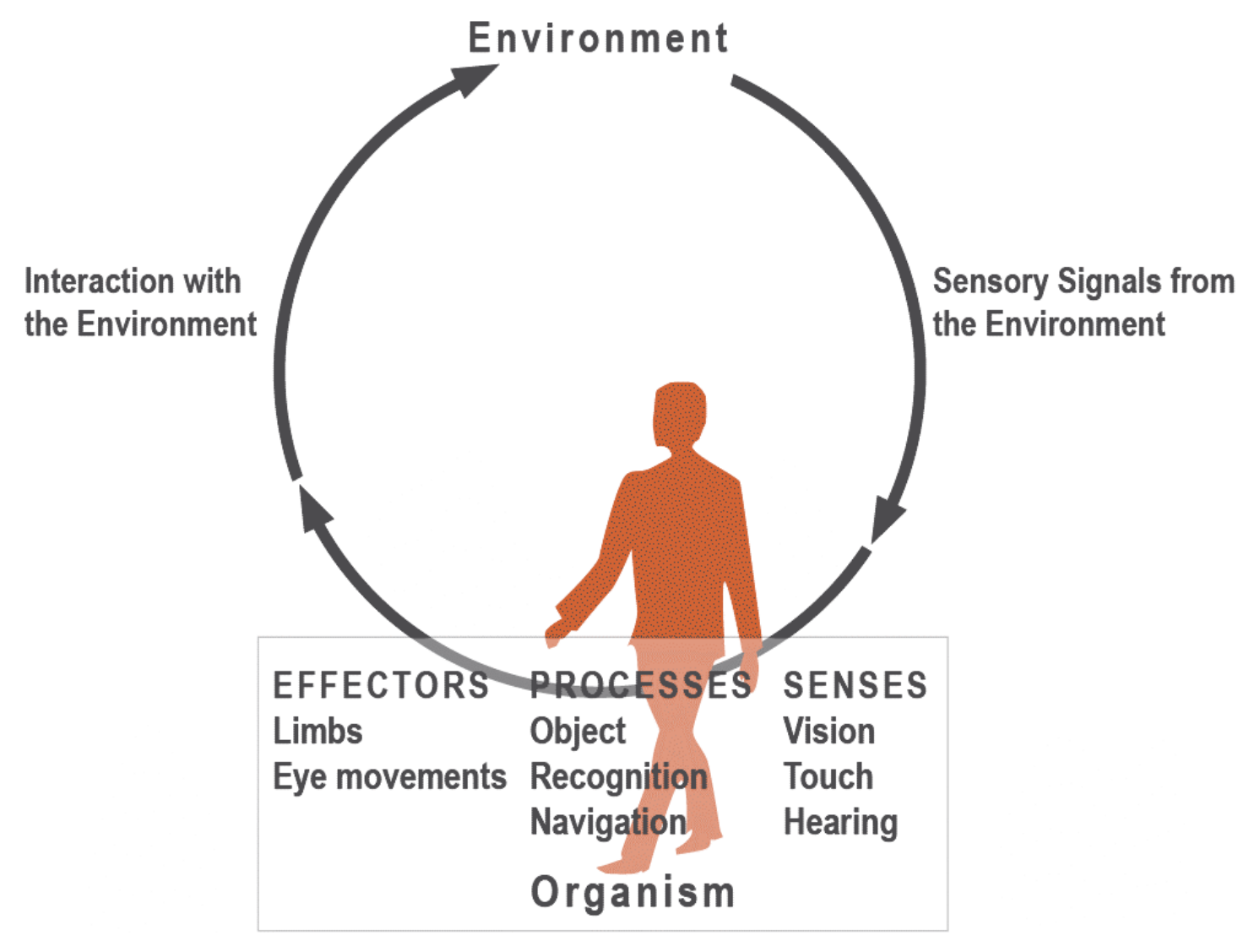




\section{Learning multi-modal Object Representations}

Natale, Rao, Sandini \& Wallraven [CogVis Project, 2004],

Wallraven \& Bülthoff [Object Recognition, Attention, Action, 2007]

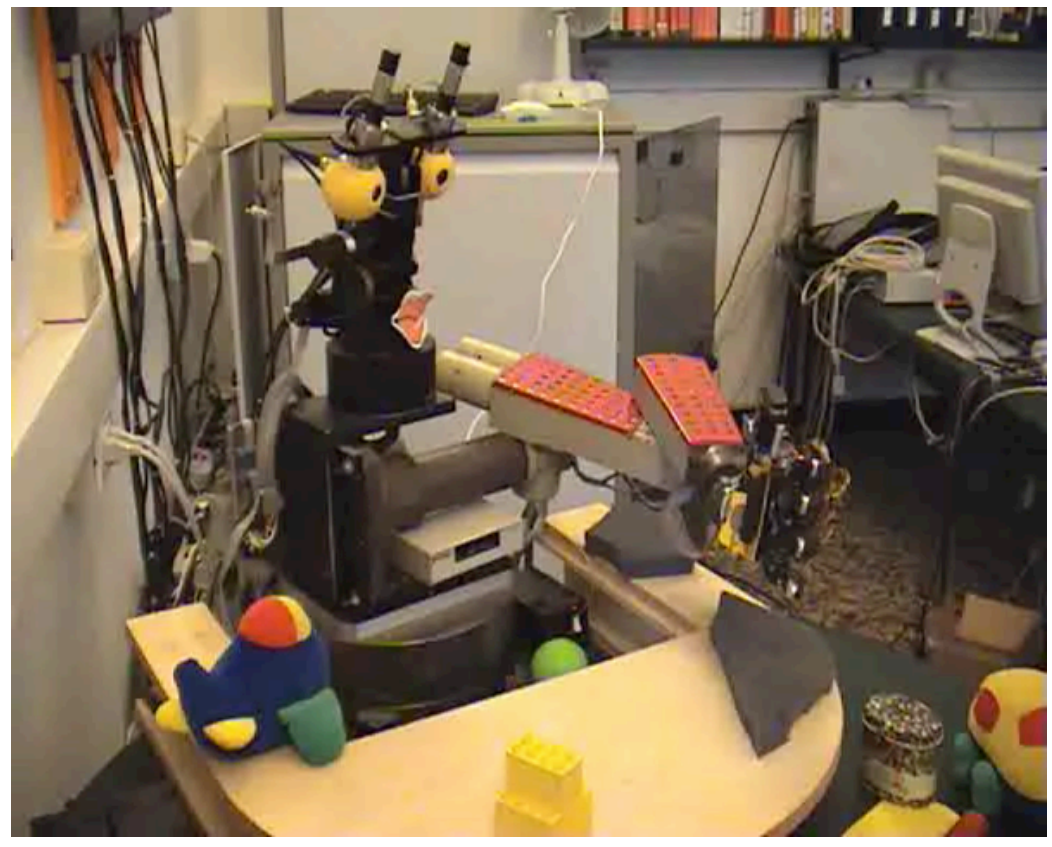

"How can

Proprioception, Vision and Active Control make object recognition more robust?" 


\section{Learning multi-modal Object Representations}

Natale, Rao, Sandini \& Wallraven [CogVis Project, 2004],

Wallraven \& Bülthoff [Object Recognition, Attention, Action, 2007]

- A robot with stereo-cameras, an arm equipped with proprioceptive sensors (LiraLab Baby-Bot)

- A simple, view-based visual recognition framework that learns object representations from image sequences

(Wallraven \& Bülthoff [CVPR, 2001])

- Coupling of proprioceptive information (joint angles) with views for learning and recognition

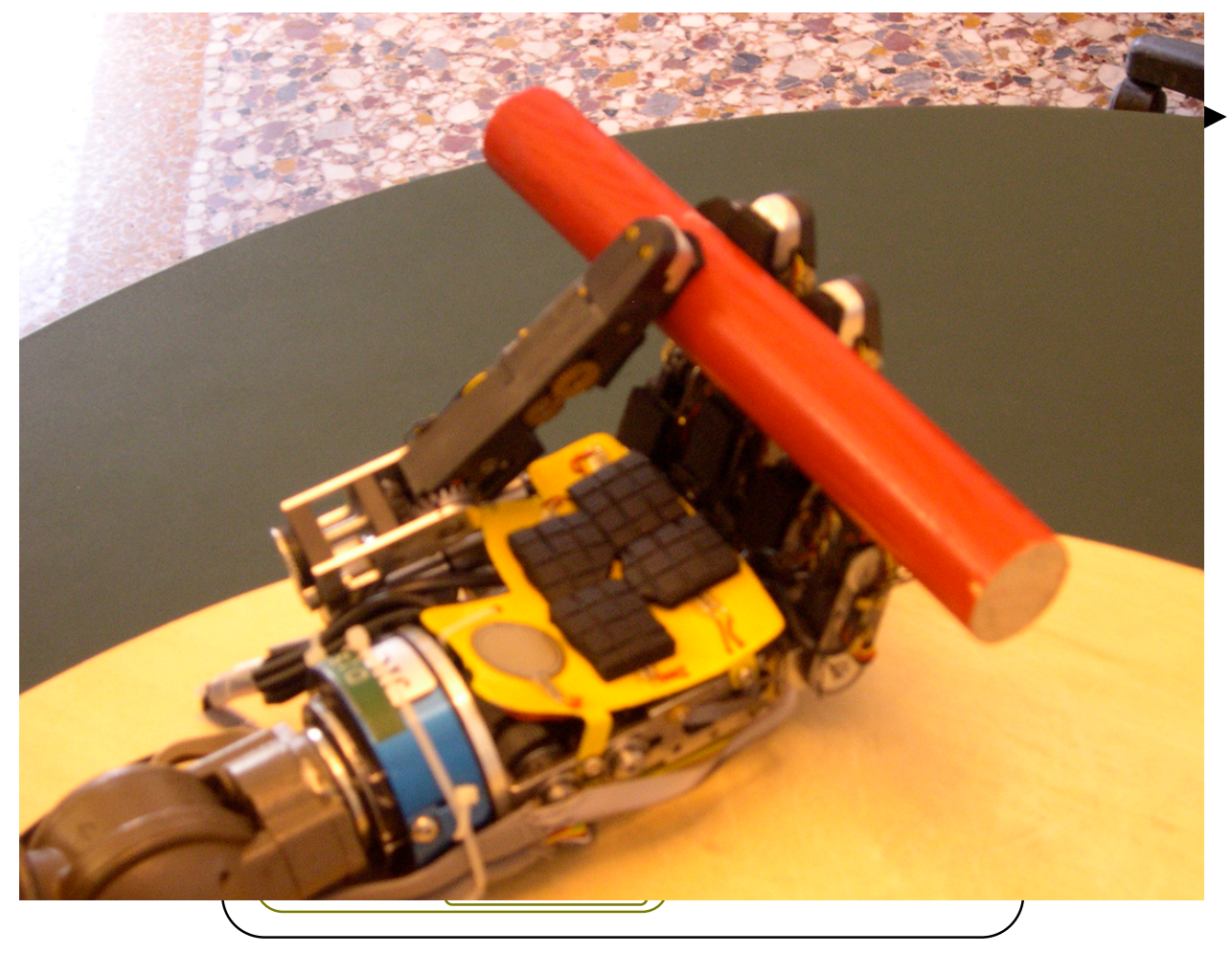




\section{Learning multi-modal Object Representations}

Natale, Rao, Sandini \& Wallraven [CogVis Project, 2004],

Wallraven \& Bülthoff [Object Recognition, Attention, Action, 2007]

- Robot performs explorative motor-program for any given object to learn the multi-sensory representation

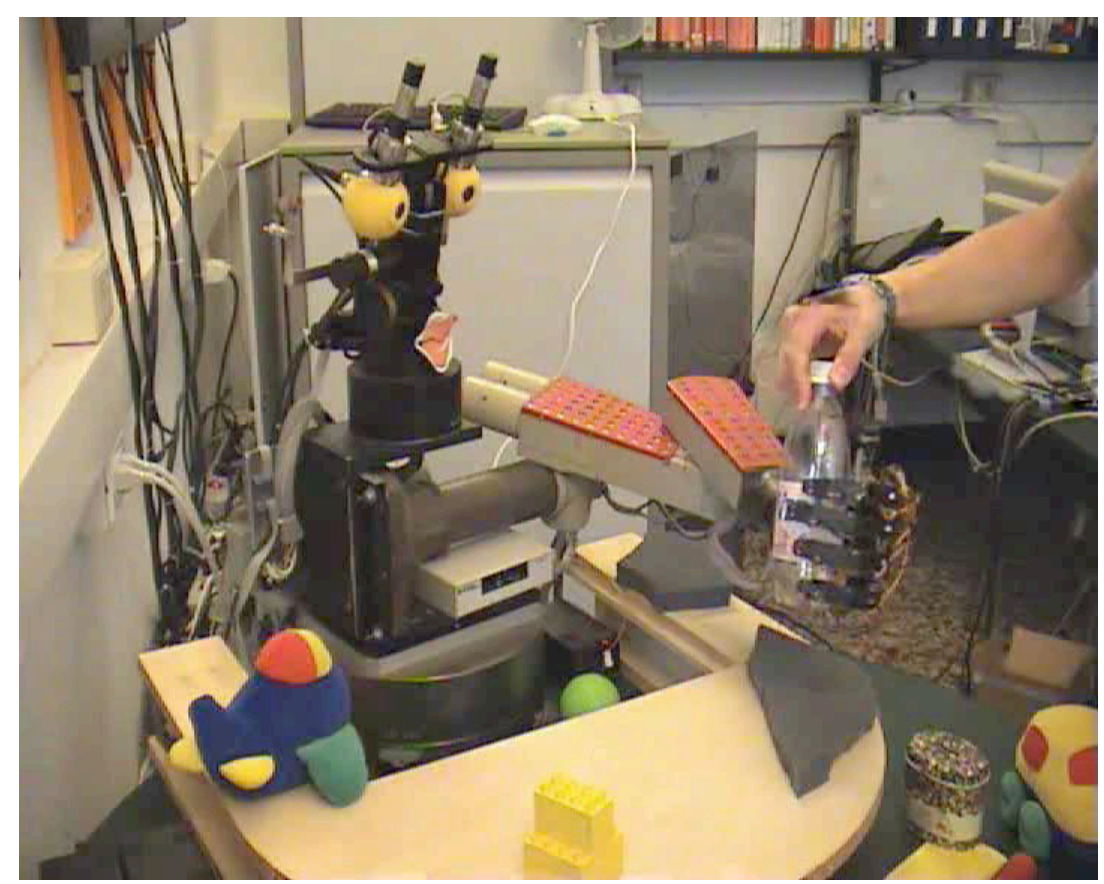

External View

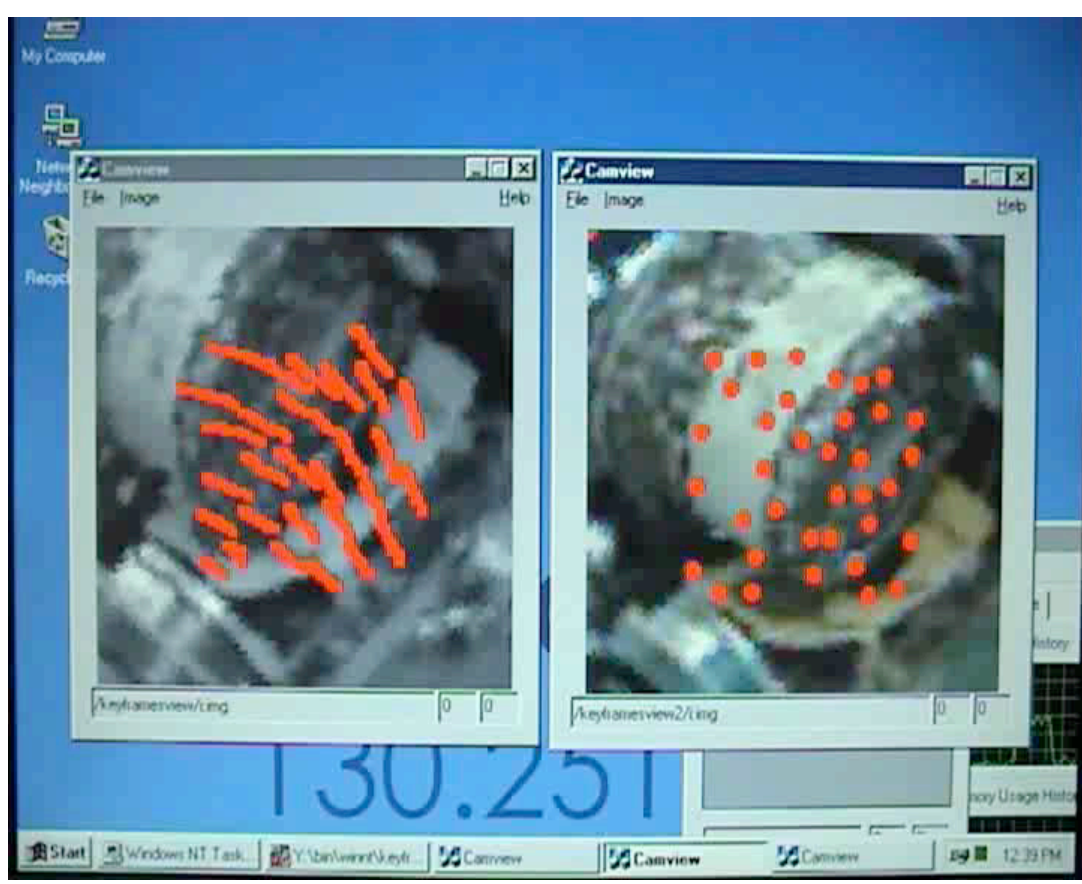

Keyframes

Tracking 


\section{Learning multi-modal Object Representations}

Natale, Rao, Sandini \& Wallraven [CogVis Project, 2004],

Wallraven \& Bülthoff [Object Recognition, Attention, Action, 2007]

- Visual matching is sufficient to predict the best model but is not very discriminatory

- Multi-modal matching profile is more "sharply tuned"

- The integration of proprioceptive information adds viewer-centered 3D information

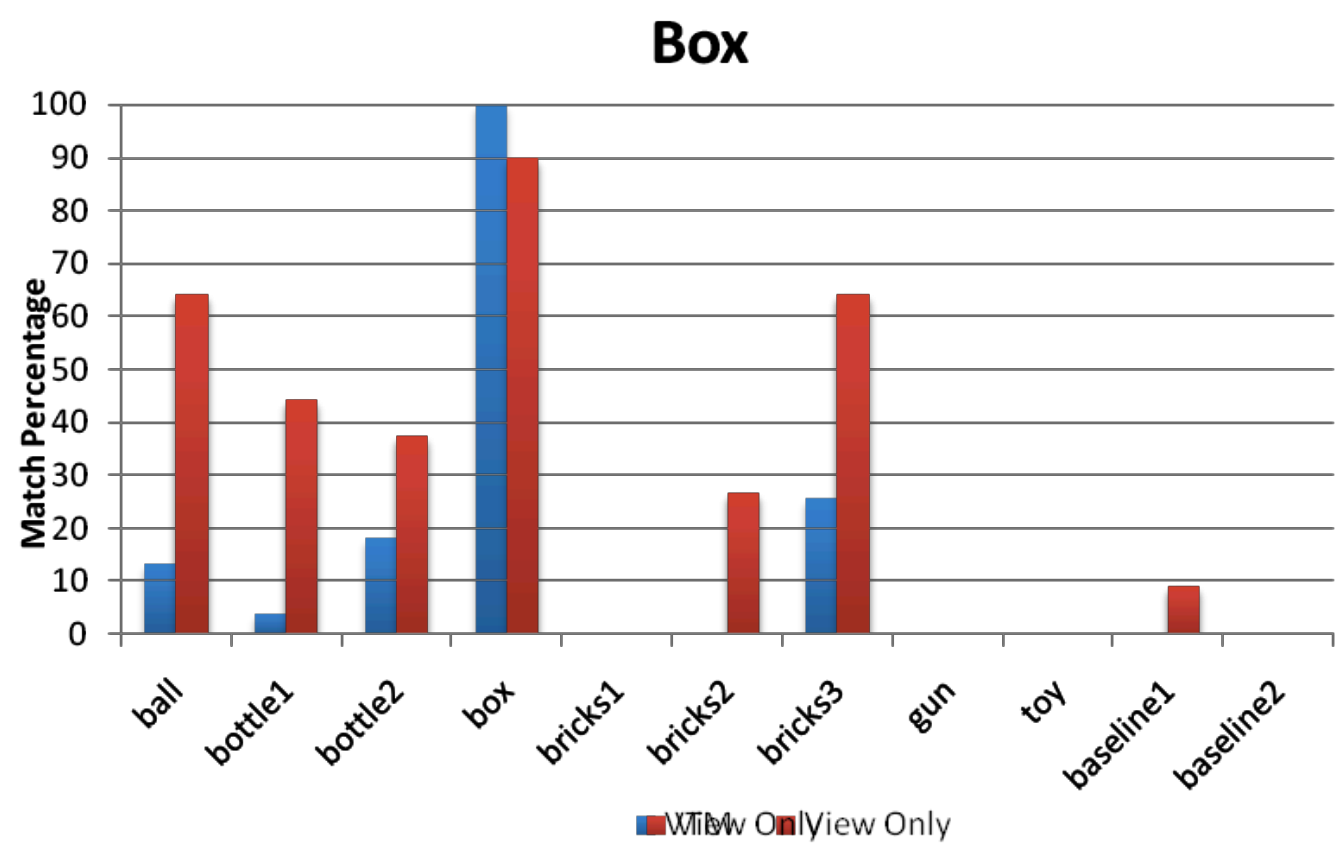




\section{Quick summary (Sensory Integration)}

- Object representations can incorporate multi-sensory information

- Common representation for vision and haptics (?)

- Cross-modal transfer between vision and haptics Newell, F., M. O. Ernst, B. S. Tjan and H. H. Bülthoff Psychological Science [2001]

- Exploitation of common representation to develop more efficient object learning and recognition algorithms for embodied agents 


\section{Some open questions}

- Computer vision

- Can we go beyond image fragments ("bags of words")?

- Do the current approaches scale to 1000s of categories?

- How do we incorporate other modalities?

- Computer graphics

- What is perceptual realism?

- How can we make better animations?

- Can we learn graphics?

- Perception research

- Can we come up with a quantitative model for object recognition?

- Does optimal integration hold everywhere - where does it break?

- What is the psychophysics of higher-level cognitive functions? 


\section{Challenges}

- The "Chair" challenge

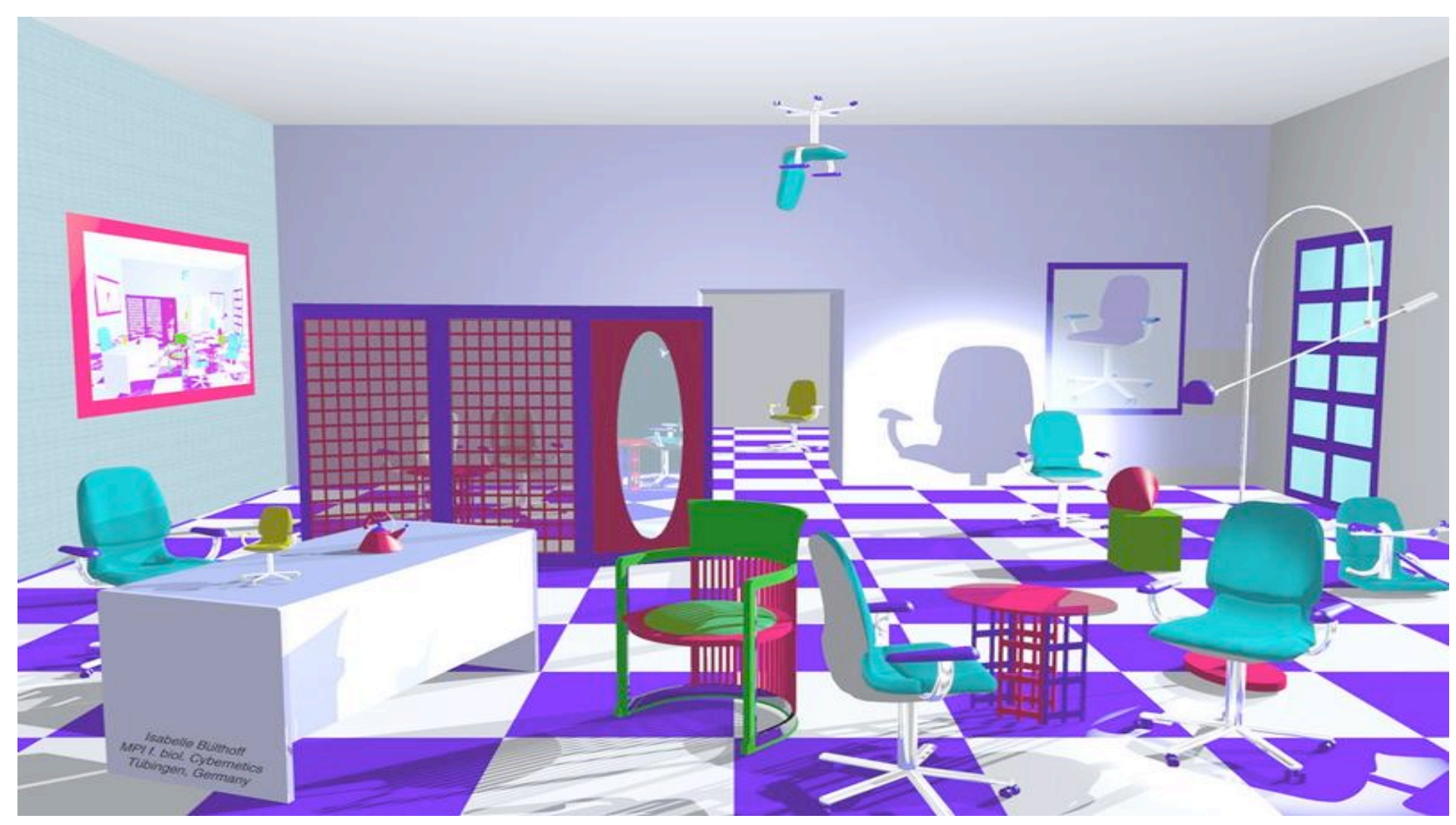




\section{Challenges}

- The "Art" challenge: build a computer vision system that learns to interpret art images

- Such a system would need to deal with abstraction
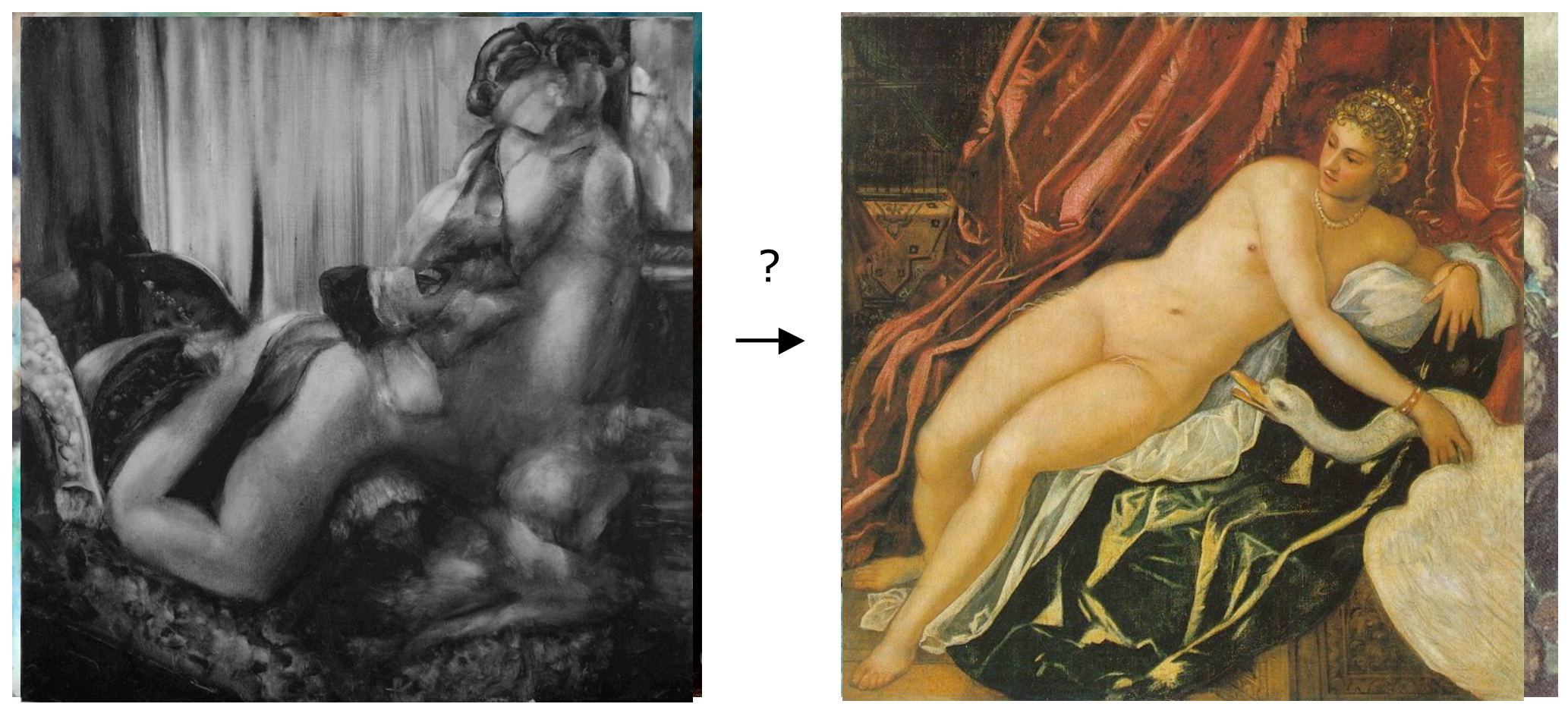

Images (c) by Robert Pepperell, see Wallraven et al. [APGV, 2007] 


\section{Challenges}

- The "Pawan Sinha" challenge

- build a computer vision system that integrates the 20 results every CV researcher should know about face recognition http://web.mit.edu/bcs/sinha/papers/20Results_2005.pdf

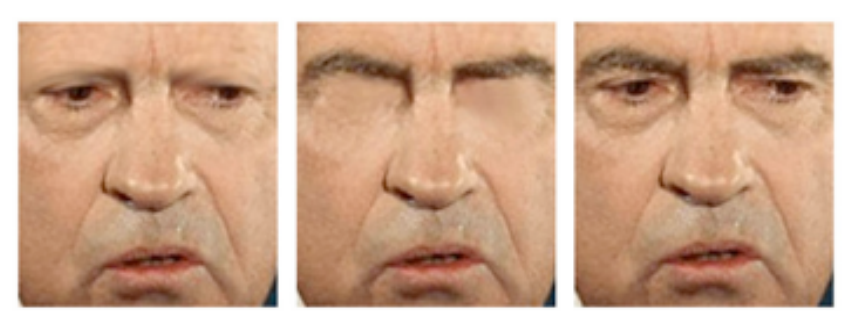

Eyebrows as important features
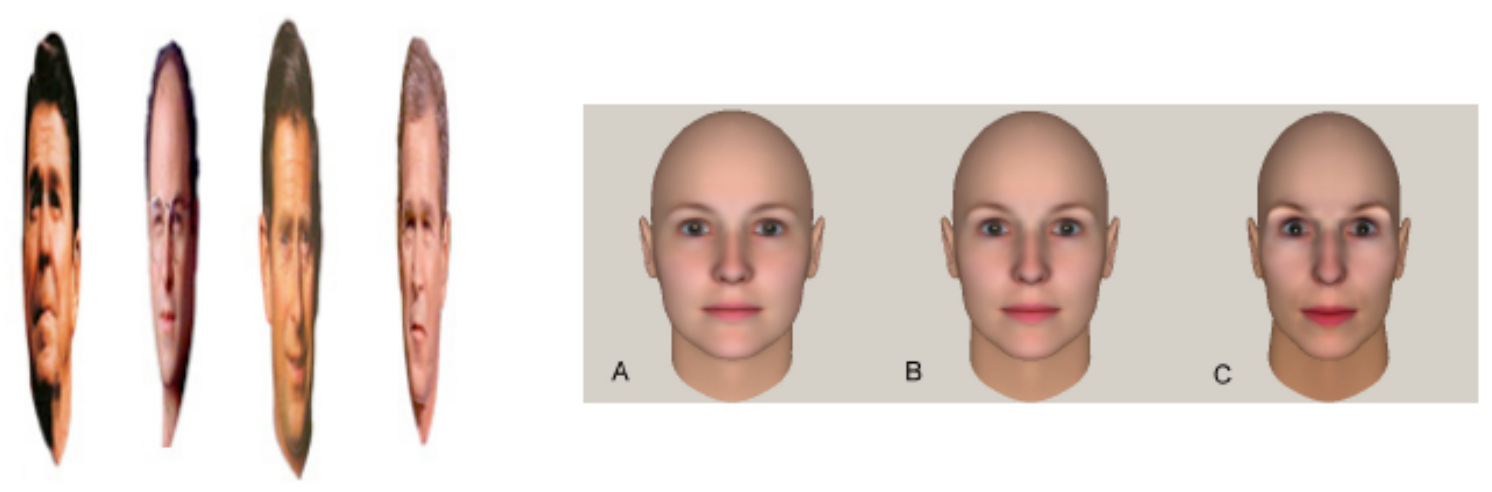

Recognition under distortions
Caricature effect for recognition 


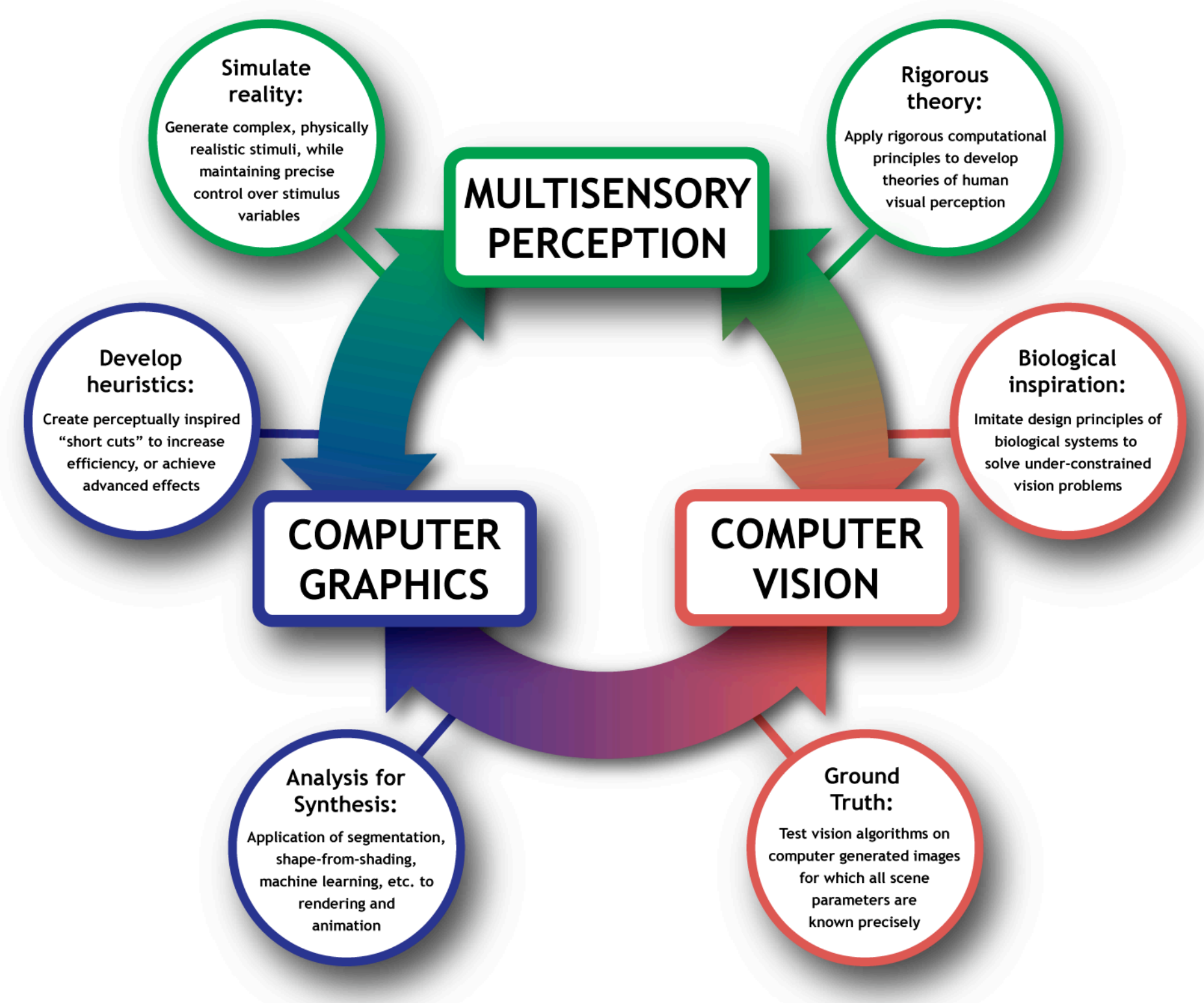

